\title{
WestVirginiaUniversity
}

THE RESEARCH REPOSITORY @ WVU

Graduate Theses, Dissertations, and Problem Reports

2016

\section{Diamond Bur Cutting Efficiency of Dental Zirconia}

\author{
Shelby Allison Alexander
}

Follow this and additional works at: https://researchrepository.wvu.edu/etd

\section{Recommended Citation}

Alexander, Shelby Allison, "Diamond Bur Cutting Efficiency of Dental Zirconia" (2016). Graduate Theses, Dissertations, and Problem Reports. 5059.

https://researchrepository.wvu.edu/etd/5059

This Thesis is protected by copyright and/or related rights. It has been brought to you by the The Research Repository @ WVU with permission from the rights-holder(s). You are free to use this Thesis in any way that is permitted by the copyright and related rights legislation that applies to your use. For other uses you must obtain permission from the rights-holder(s) directly, unless additional rights are indicated by a Creative Commons license in the record and/ or on the work itself. This Thesis has been accepted for inclusion in WVU Graduate Theses, Dissertations, and Problem Reports collection by an authorized administrator of The Research Repository @ WVU. For more information, please contact researchrepository@mail.wvu.edu. 


\title{
Diamond Bur Cutting Efficiency of Dental Zirconia \\ Shelby Allison Alexander, D.D.S.
}

Thesis submitted to the

\author{
School of Dentistry \\ At West Virginia University \\ in partial fulfillment of the requirements \\ for the degree of \\ Master of Science \\ In \\ Prosthodontics
}

\author{
Matthew S. Bryington, D.M.D., M.S. (Chair) \\ Bryan D. Dye, D.D.S., M.S. \\ Michael D. Bagby, D.D.S., Ph.D., M.S.
}

Department of Restorative Dentistry

Morgantown, West Virginia

2016

Keywords: Dental Zirconia, Diamond Bur Cutting Efficiency, Zirconia Crown Removal Copyright 2016 Shelby Alexander 


\title{
ABSTRACT \\ Diamond Bur Cutting Efficiency of Dental Zirconia
}

\author{
Shelby Allison Alexander, D.D.S
}

Objectives: To monitor the rate of diamond bur cutting through sintered yttria stabilized zirconia. Through this observation, we intend to determine which bur(s) exhibit the greatest cutting efficiency and if a decline exists over time.

Methods: Seven dental diamond burs were used in an air-turbine hand piece to cut sintered Yttria Stabilized Zirconia blocks (3Y-TZP) at a constant $0.9 \mathrm{~N}$ force for five minutes. The distance traveled was measured by a Linear Variable Differential Transformer (LVDT) and recorded per time by a data acquisition device. Time was divided into 100-second intervals for comparison. Individual effects of bur, time periods, and depth on cutting efficiency were evaluated. Combined effect of bur and time periods was also evaluated.

Results: Statistically significant differences were found between bur, time period and depth on bur cutting efficiency of $3 \mathrm{Y}-\mathrm{TZP}$. Combined effect of bur and time period did not demonstrate a significant difference. Burs with medium, coarse, and super coarse diamond particles exhibited greater cutting efficiency of sintered $3 \mathrm{Y}$-TZP than burs with fine diamond particles. Cutting efficiency of all burs was significantly greater within the initial 100 second cutting time period.

Conclusions: For removal of 3Y-TZP crowns intraorally via sectioning, the results of this study suggest it is best to use a diamond bur with medium, coarse or super coarse particle grit size and limit its use to 100 seconds in order to maximize cutting efficiency. The super coarse diamond had the greatest cutting efficiency throughout the 5-minute evaluation period. Future studies are needed to evaluate cutting efficiency after low temperature degradation (LTD) and surface manipulations on $3 \mathrm{Y}-\mathrm{TZP}$ in order to duplicate exposure to the oral environment, and expanding the testing time beyond 5 minutes to determine if and when a second significant difference in cutting efficiency occurs. 


\section{DEDICATION}

I would like to dedicate this paper to my father who also functioned as the project engineer, John E. Faltot PE. Without your support and guidance much of my accomplishments in life would not have come to fruition, especially this project. 


\section{ACKNOWLEGEMENTS}

I would like to thank the following individuals for providing much needed guidance during my postdoctoral training:

Dr. Matthew S. Bryington, Thank you for teaching me the art and science of Prosthodontics. You have been the best mentor anyone can ask for in advanced education. Your never-ending support and guidance will never be forgotten.

Dr. Bryan D. Dye, Thank you for being my role model in dentistry and exemplifying the knowledge and skill set which can be attained from hard work and dedication. You are the reason I wanted to become a Prosthodontist and I strive to produce quality care up to your standard of excellence.

Dr. Michael Bagby, Thank you for your support and guidance through this process especially stimulating my critical thinking of experimental design and controlling for error. I greatly appreciate your material science expertise and never ending dedication to education.

Dr. Gerald Hobbs, Thank you for your statistical expertise.

I would also like to thank the following for their generous donation of the materials for the project:

Todd Warner and Ivoclar Vivadent for providing the sintered zirconia blocks.

Joe Rittler and Brasseler USA for providing the Brasseler burs. 


\section{TABLE OF CONTENTS}

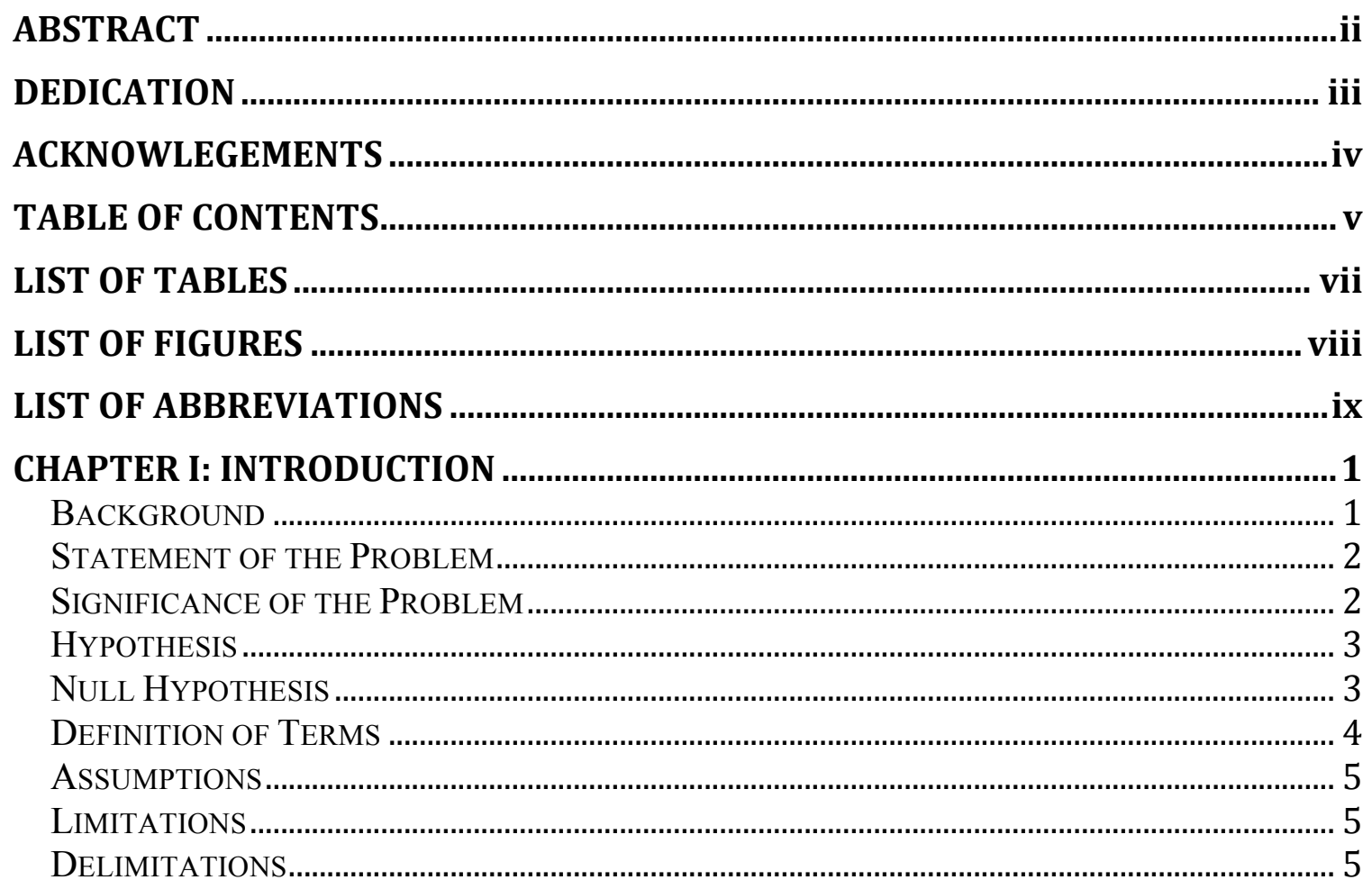

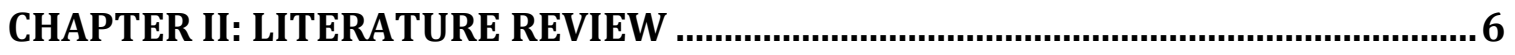

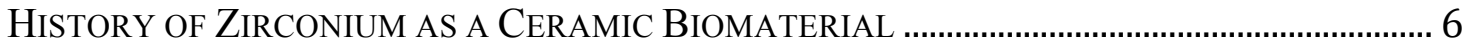

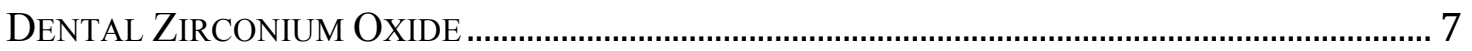

ZIRCONIA VERSUS METAL-CERAMIC RESTORATIONS.................................................................. 8

INDICATIONS FOR PLACEMENT/REPLACEMENT OF CROWNS AND FDPS .............................12

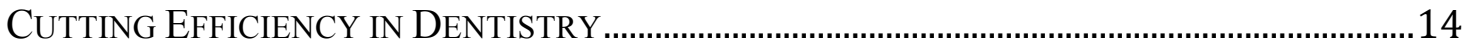

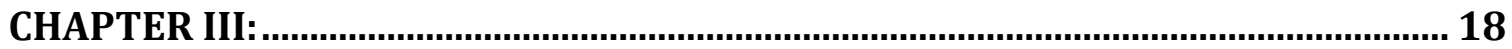

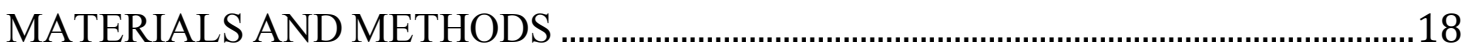

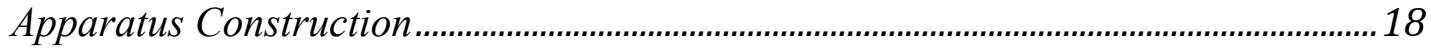

Data Recording Instrumentation and Configuration .................................................... 19

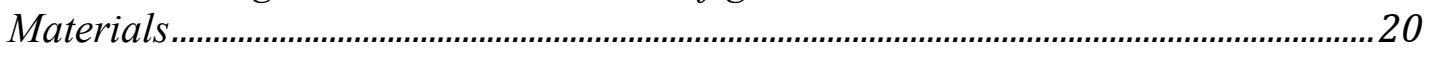

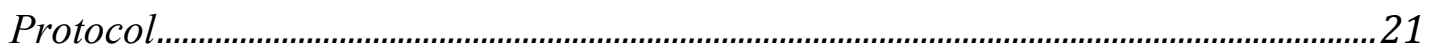

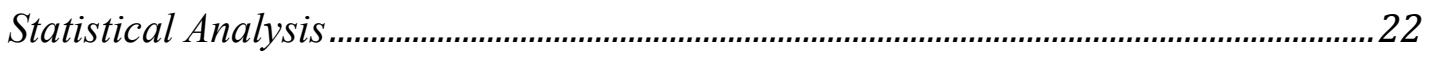

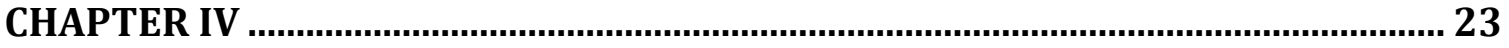

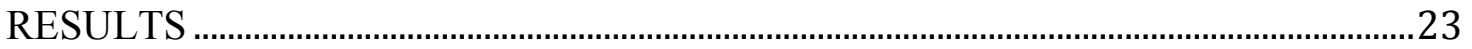

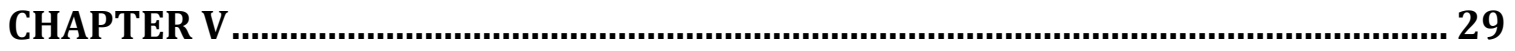

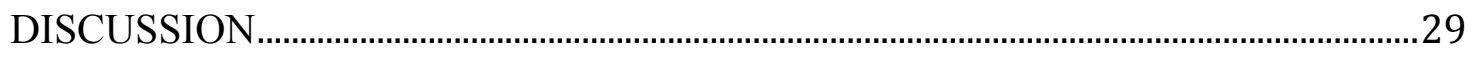




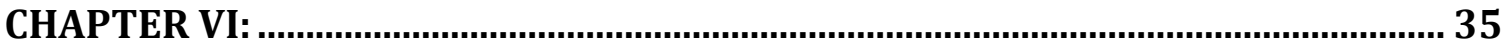

SUMMARY

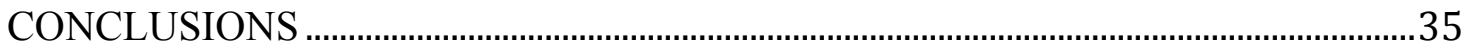

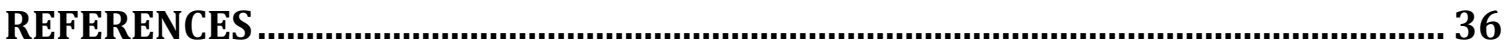

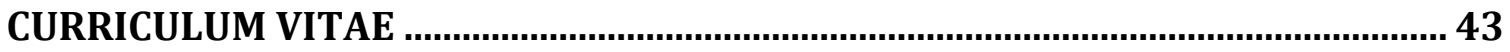




\section{LIST OF TABLES}

Table 1. Bur Characteristics.

Table 2. Mixed Effects ANOVA.

Table 3. Tukey-Kramer HSD defining significance between burs.

Table 4. Tukey-Kramer HSD defining significance between periods.

Table 5. Fisher LSD defining differences between burs in Period 1. 


\section{LIST OF FIGURES}

Figure 1. Experiment Apparatus

Figure 2. Experiment Assembly

Figure 3. Cutting efficiency of test burs over 5 minutes

Figure 4. Mean distance cut by each bur group after 5 minutes

Figure 5. Cutting efficiency average for bur groups in each Period

Figure 6. Cutting efficiency of test burs in Period 1

Figure 7. Oneway ANOVA depicting cutting efficiency in Period 1 


\section{LIST OF ABBREVIATIONS}

3Y-TZP- Yttria-Stablilized Tetragonal Zirconia Polycrystals

CAD/CAM- Computer aided design, computer assisted manufacturing

FDP- Fixed Dental Prosthesis

LTD- Low-temperature degradation

PSZ- Partially Stabilized Zirconia

ZTA- Zirconia Stabilized Alumina 


\section{CHAPTER I: INTRODUCTION}

\section{Background}

The dental procedure of replacing missing teeth, tooth structure or providing additional support for endodontically treated teeth by means of the full coverage dental restoration or fixed dental prosthesis (FDP) is a common practice. The materials available for such restorations have been classified based on strength and fracture resistance. Historically, cast metal and metal ceramic restorations were indicated in posterior regions and all ceramic restorations were strictly limited to use in the anterior. However, a dynamic shift in the utilization of ceramic restorations has occurred due to the recent advancements in material science.

The move to use ceramic crowns in place of the traditional cast metal or metal ceramic crowns is one of the fastest, widespread changes that dentistry has seen in its history. The increased demand for esthetics, introduction of Computer aided Design and Computer aided Manufacturing(CAD/CAM), and the rise in gold costs has paved the way for development of higher strength ceramics such as yttria-stablilized tetragonal zirconia polycrystal ceramics (3Y-TZP). Due to its favorable qualities of increased fracture resistance, esthetics, biocompatibility, decreased time for fabrication and low cost, $3 \mathrm{Y}$ TZP has become a go-to restoration in anterior and posterior regions for single crowns, fixed dental prosthesis and full arch restorations.

Despite the advancements in dental diagnosis, treatment, and materials, restoration failure still occurs. Failures due to crown fracture, loss of retention, recurrent decay, or loss of pulp vitality are just some of the many reasons that may necessitate removal and replacement of a prosthesis. In situations where replacement is necessary, the removal of such prostheses should be conservative and efficient.

A common way to remove a previously placed crown is by placing a slot in the crown, extending it onto multiple exposed surfaces, then torqueing the halves using an instrument in the slot. Dental zirconia has posed a problem in removal in this way due to its high flexural strength and hardness. Attempts to section dental zirconia often result in 
destroying multiple burs, loss of chair time, and frustration by the dentist and patient.

\section{Statement of the Problem}

Does the type of diamond dental bur affect the rate of cutting dental zirconia restorations?

\section{Significance of the Problem}

A marked increase in the clinical application of dental zirconia has occurred over the last decade. Due to its increase in prevalence, the dental literature has been flooded with reports validating its use and demonstrating ways to improve its function and application as a dental ceramic. Zirconia restorations have been recommended due to their esthetics, biocompatibility, and favorable biomechanical properties that allow their use in clinically challenging situations. In addition, zirconia has favorable industry characteristics such as decreased fabrication time, lower costs, and a low number of remakes due to fracture. Monolithic zirconia restorations are recommended as a viable solution for bruxers due to their high strength and fracture resistance ${ }^{1}$

A limited number of conflicting articles are available to describe the best instrument or technique for removal of zirconia restorations. A Clinician's Report proposed a technique utilizing a fine grit diamond, high water spray with a light sawing motion to remove lithium disilicate or zirconia crowns. They also stated that this technique requires four slots, four diamond burs and four minutes to complete ${ }^{2}$. Due to the perceived difficulty in zirconia crown removal, several studies have evaluated which dental burs have the greatest cutting efficiency. In 2011, Ohkuma et al. evaluated diamond grit size as it relates to zirconia cutting efficiency and found that even with light touch a larger diamond grit size of $200 \mu \mathrm{m}$ was more efficient than $100 \mu \mathrm{m}^{3}$. This is further supported by Nakamura and colleagues who concluded that diamond burs with super coarse grains $(300 \mu \mathrm{m})$ were most effective ${ }^{4}$. Interestingly, these three reports recommended limiting the duration of each bur's use due to pullout, chipping, and wear

of the diamond particles, which decreases the bur's cutting ability ${ }^{2-4}$. These studies have failed to evaluate cutting efficiency with a dynamic measure over time and provided 
insufficient data for clinical application due the limited number of burs used in comparison for zirconia crown removal.

\section{Hypothesis}

The type of diamond bur will have an effect on cutting efficiency measured by rate of 3 Y-TZP cutting within 5 minutes. The type of diamond bur will also affect the time at which each bur's cutting efficiency declines.

\section{Null Hypothesis}

Bur type will not have an effect on cutting efficiency over 5 minutes. The bur type will not affect the bur's cutting efficiency rate of decline. 


\section{Definition of Terms}

Alloying element- metallic or non-metallic elements added to or retained by a pure metal for the purpose of giving that metal special properties ${ }^{5}$.

Bruxism: the parafunctional grinding of teeth ${ }^{5}$.

CAD/CAM: acronym for Computer Aided Design, Computer Aided Machining. An alternative fabrication process which electronically or digitally records surface coordinates to allow digital design of prosthesis. The coordinates then can be used to mill or grind by computer control the prosthesis from a solid block of the chosen material $^{6}$.

All-ceramic restoration: a ceramic fixed dental prosthesis that restores a clinical crown without a supporting metal framework ${ }^{5}$.

Crown- an artificial replacement that restores missing tooth structure by surrounding part or all of the remaining structure with a material such as cast metal, porcelain, or a combination of materials such as metal and porcelain ${ }^{5}$.

Fixed dental prosthesis- any dental prosthesis that is luted, screwed, or mechanically attached or otherwise securely retained to natural teeth, tooth roots, and/or dental implant abutments that furnish the primary support for the dental prosthesis ${ }^{5}$.

Fracture toughness- is a mechanical property that describes the resistance of brittle materials to the catastrophic propagation of flaws under an applied stress. It is given in units of stress times the square root of crack length $\left(\mathrm{MPa} \cdot \mathrm{m}^{1 / 2}\right)^{6}$.

Metal ceramic restoration- a tooth or implant retained fixed dental prosthesis that uses a metal substructure upon which a ceramic veneer is fused ${ }^{5}$.

Modulus of elasticity (Young's)- describes the relative stiffness or rigidity in a material $^{6}$. It is a ratio of stress to strain. As the modulus of elasticity rises, the material becomes more rigid ${ }^{5}$. 
Sinter- to cause to be come a coherent mass by heating without melting ${ }^{5}$.

Transformation toughening- the resistance created against an advancing crack due to the production of compressive stress in partially stabilized zirconia (PSZ). This occurs when the crystalline form of zirconia transforms from the metastable tetragonal phase to the stable monoclinic phase ${ }^{6}$.

\section{Assumptions}

1. It is assumed the sintered 3Y-TZP blocks are homogenous and structurally identical to sintered 3Y-TZP crowns.

2. It is assumed each bur type is consistent with the manufacturer's defined dimensions and diamond particle size.

\section{Limitations}

1. An air turbine handpiece was used for the study, which limited the ability to have a constant RPM by controlling torque. Use of electric handpiece was not possible due to it over heating at 90 seconds.

2. Coolant flow rate of $16 \mathrm{ml} / \mathrm{min}$ was used due to the dental unit's limited adjustment ability.

\section{Delimitations}

1. Limited cutting time to 5 minutes due to zirconia block length.

2. Limited test burs based on shank design; included only those that were parallel sided and round-ended. 


\section{Chapter II: LITERATURE REVIEW}

\section{History of Zirconium as a Ceramic Biomaterial}

Zirconium is an elemental metal with an atomic number of 40 ; its name is derived from the Arabic term Zargon meaning golden in color. Zirconia $\left(\mathrm{ZrO}_{2}\right)$ is the crystalline dioxide of zirconium which was identified in 1789 by a German chemist and was utilized as a pigment for ceramics ${ }^{7,8}$. Zirconia has been observed to have unique temperaturedependent phase transformations. Unalloyed zirconia can assume three crystalline forms depending on the temperature; monoclinic (room temperature to $1170^{\circ} \mathrm{C}$ ), tetragonal $\left(1170{ }^{\circ} \mathrm{C} \text { to } 2370^{\circ} \mathrm{C} \text { ), and cubic (above } 2370^{\circ} \mathrm{C}\right)^{7-9}$.

Zirconia's unique mechanical properties are the result of a volumetric increase when transforming from tetragonal to monoclinic phase during cooling. Catastrophic failure of the material can occur during transformation in pure zirconia, but can be stabilized with the addition of other metallic oxides such as $\mathrm{CaO}, \mathrm{MgO}, \mathrm{CeO}_{2}$, or $\mathrm{Y}_{2} \mathrm{O}_{3}$ to create partially stabilized zirconia (PSZ). The supplementation of other metal oxides allows a partial retention of the tetragonal structure at room temperature. This process results in transformation toughening 9 .

Crack formation is a process that occurs when stress is applied to the surface of PSZ. The energy created by the stress on the forming crack stimulates a phase transformation from the tetragonal to the monoclinic crystalline structure in the material leading the fracture. This phase shift results in volumetric expansion which places pressure on the crack, making it harder to propagate and contributes to PSZ's toughness and fracture resistance ${ }^{7-10}$. The discovery of PSZ's transformation toughening expanded its use to the machining industry due to its mechanical properties such as favorable chemical and dimensional stability, mechanical strength and toughness, and Young's modulus similar to stainless steel alloys ${ }^{7,8}$.

In 1969, orthopedics research and development began to look at zirconia as a potential replacement for titanium and alumina ball heads in Total Hip Replacements ${ }^{7}$. Such use as a biomaterial launched studies to investigate its biocompatibility and 
potential cytotoxic properties. In vitro studies since the 1990s have demonstrated the biocompatibility of zirconia when implanted into bone or muscles of various animal species. Those studies have confirmed $\mathrm{ZrO}_{2}$ is not only non-cytotoxic, but creates less inflammatory infiltrate, microvessel density, and vascular endothelial growth factor expression than titanium ${ }^{7,11}$.

Some caution was given to the utilization of $\mathrm{ZrO}_{2}$ in an acidic environment, such as the oral cavity, due to the release of zirconium and yttrium ions ${ }^{8}$. Further investigations have determined the ion release was due to zirconium hydroxide, which is eliminated after sintering and allowing the safe application of solid zirconia's use in dentistry ${ }^{7}$.

\section{Dental Zirconium Oxide}

Although PSZ had been used as a ceramic biomaterial in medicine starting in the 1970s, its utilization in dentistry did not start until the early 1990s and has since been limited to two zirconia-containing ceramic systems ${ }^{12}$. They include zirconia-toughened alumina (ZTA) and yttrium stabilized tetragonal zirconia polycrystals (3Y-TZP) ${ }^{9}$. Due to its $8-11 \%$ porosity and decreased mechanical properties, ZTA is much less commonly used compared to $3 \mathrm{Y}-\mathrm{TZP}^{9}$. $3 \mathrm{Y}$-TZP gets its name from the addition of 3 mole $\% \mathrm{Y}_{2} \mathrm{O}_{3}$ which stabilizes the tetragonal structure at room temperature ${ }^{11}$. 3Y-TZP, with a fracture toughness from 5 to $10 \mathrm{MPa} \cdot \mathrm{m}^{1 / 2}$ and a flexural strength of $900-1400 \mathrm{MPa}$, exhibits better fracture resistance compared to all other dental ceramics ${ }^{13,14}$.

The stability of $3 \mathrm{Y}-\mathrm{TZP}$ at room temperature and maintaining the desired tetragonal phase is a delicate science. Particular attention must be paid not only to the composition, size, and shape of zirconia particles, but also the type and amount of metal oxide, final heat processing, and interphase interactions within the zirconia ${ }^{13}$. For example, above a critical grain size 3Y-TZP becomes less stable and more susceptible to spontaneous tetragonal to monoclinic transformation. Below a certain grain size phase transformation is impossible, eliminating transformation toughening and resulting in greatly decreased fracture resistance. The sintering process has been shown to affect the 
grain size, with higher temperatures and longer sintering times resulting in larger grain size $^{9,15}$.

The use of 3Y-TZP in dentistry has been made possible by CAD/CAM technology and a milling machine's ability to cut a zirconia block into the desired final form. Milling of zirconia is routinely done in one of three block states: green, presintered and fully sintered. The green state describes the zirconia in a chalk-like form by which the 3Y-TZP powder has been added to a binder and condensed by cold isostatic pressing into the form of a block ${ }^{9}$. This block can then be presintered, where heat is applied slowly, eliminating the binder and enhancing the hardness and machinability ${ }^{9}$. A final heat treatment develops the definitive mechanical properties and dimension of the $3 \mathrm{Y}$ TZP .

The fabrication of a full contour restoration or coping can be completed in two ways using $\mathrm{CAD} / \mathrm{CAM}$ technology: soft milling or hard milling. Soft milling involves either machining an enlarged form of the final product from a homogenous green block or a partially sintered block. $3 \mathrm{Y}$-TZP shrinks $20-25 \%$ during the sintering process, this shrinkage must be accurately predicted and accounted for during the initial milling to assure an accurate fit ${ }^{7,10,16,17}$. Hard milling is machining of the final product from a fully sintered zirconia block. The drawback of hard milling is the wear and tear on the milling tools and potential for incorporation of unwanted defects in the zirconia during machining ${ }^{18}$.

\section{Zirconia versus Metal-ceramic Restorations}

In 2014, Gordon Christensen published an article describing the trend from using metal-ceramic to all ceramics as "one of the fastest and most significant paradigm changes in the history of dentistry" ${ }^{19}$. He published data from a commercial laboratory which found a $335 \%$ increase in all ceramic restorations compared to a $386 \%$ decrease of metal-ceramic restorations from 2007 to $2013^{19}$. At this time, long term evidence is not available in the literature to support the broad-spectrum use of all ceramic restorations, however short-term studies have shown positive results and tentatively support its 
growing use $\mathrm{e}^{20-23}$. Studies are available which compare zirconia and metal ceramic restorations in regards to marginal fit and accuracy of CAD/CAM technology, esthetics, failure and complication rates, maintenance of strength at reduced thickness and biocompatibility ${ }^{23-39}$.

Common dental indications for 3Y-TZP include anterior and posterior single unit crowns and fixed dental prosthesis. Clinical acceptability for such uses has been supported by studies evaluating marginal fit and internal adaptation, not only to evaluate 3Y-TZP as a dental ceramic but also the accuracy of the CAD/CAM fabrication process $^{24,26,27,29,31}$. Three studies evaluated the marginal discrepancies of three 3Y-TZP systems using $\mathrm{CAD} / \mathrm{CAM}$ technology for fabrication compared to metal-ceramic fabricated by the lost wax casting technique. Not only were the 3Y-TZP systems within the $100 \mu \mathrm{m}$ clinical acceptable range for marginal adaption, but the discrepancies were also lower than the metal ceramic group ${ }^{24,26,27}$. This is further supported by the findings of Vigolo and Fonzi and Lins et al., which examined three 3Y-TZP systems evaluating marginal fit before and after porcelain firing cycles of 4-unit FDPs. They reported the firing cycles did not affect the marginal fit and showed the marginal adaptation of FDPs to be within a clinically acceptable range ${ }^{29}$. Furthermore, Lins et al. evaluated the marginal and internal fit of $\mathrm{CAD} / \mathrm{CAM}$ zirconia copings and found all were within clinically acceptable ranges ${ }^{31}$. These studies demonstrate the viability of CAD/CAM technology for fabricating 3Y-TZP single crowns or fixed dental prosthesis with clinically acceptable marginal fit and internal adaptation.

As stated earlier, 3Y-TZP restorations can be fabricated as monolithic restorations or as a coping to which ceramic is applied to enhance esthetics. The decision of which to fabricate is dependent on the desired strength and esthetics of the final restoration. A monolithic 3Y-TZP crown maintains its mechanical properties and like metal-ceramic restorations, has significant limitations in esthetics due to its opacity. The addition of ceramic to either a $3 \mathrm{Y}-\mathrm{TZP}$ or metal coping increases the esthetics of the restoration but subjects the restoration to fracture based on the mechanical properties inherent to the ceramic used and the strength of the ceramic to $3 \mathrm{Y}-\mathrm{TZP}$ interface. Unlike metal ceramic, zirconia copings have an advantage in being white in color. In situations of gingival 
recession or thin gingiva, metal ceramic restorations often present with a gray hue at the margin, which is not present in similar zirconia restorations. Such situations make zirconia more desirable in highly esthetic areas that demand high core strength.

Multiple studies have reported the survival and complication rates of $3 \mathrm{Y}$-TZP crowns or FDPs compared to metal ceramic. A 2013 evaluation by Koeing et al. reported risk factors for failure of zirconia-based restorations. The reported success rate was $81.6 \%$ after a mean observation period of 3.5 years, where fracture of the veneering ceramic was the most common complication ${ }^{32}$. Chipping and fracture of the veneering ceramic for 3Y-TZP was also reported by Sailer et al. and Pjetursson et al. in a 2015 systematic review of the literature. They reported the 5 year complication rate of veneer ceramic chipping or fracture of single unit crowns and multiple unit fixed restorations to be $6.3 \%$ and $14.5 \%$ respectively ${ }^{23,33}$. Based on these reports, chipping and fracture of the veneering ceramic seems to be a limiting factor to the survival rates of 3Y-TZP single crowns and multiple unit FDPs.

In light of the apparent evidence of increased fracture of the veneered ceramic on 3Y-TZP compared to metal ceramic, further studies were undertaken to determine the nature and cause of the fractures. Two theories were developed: the coefficient of thermal expansion between the materials was not compatible or the framework design was insufficient to provide adequate support to the veneered ceramic. It was found in multiple reports that using a veneering ceramic with a closer coefficient of thermal expansion to $3 \mathrm{Y}-\mathrm{TZP}$ in conjunction with a slower cooling cycle after the last glaze firing minimized the thermal residual stress within the veneer and delays failure of the zirconiaveneer crowns $^{30,34-36}$. One report published 5-year results comparing the survival of metal ceramic crowns to zirconia-veneered crowns fabricated by controlling the coping design and allowing for slower cooling of the veneer ceramic. They found no significant differences in the incidence of ceramic fracture between metal ceramic crowns and ceramic-veneered zirconia with estimated cumulative five-year survival rates of $97.6 \%$ and $94 \%$ respectively ${ }^{28}$. In support of those findings, a systematic review in 2014 by Larsson and Wennerberg evaluated the clinical success of implant and tooth supported zirconia based single crowns and found cumulative 5-year survival rates of $97.1 \%$ and 
95.9\% respectively. Given that the five-year survival rate of metal ceramic restorations to exceed $95 \%$, they concluded the survival of ceramic veneered zirconia and metal ceramic restorations to be comparable. Although ceramic fracture and chipping has been seen as a common complication of 3Y-TZP veneered crowns, the evidence supplied by Rinke et al. and Larsson and Wennerberg attributes this to inappropriate ceramic selection and firing when using $3 \mathrm{Y}-\mathrm{TZP}$ as the coping. Controlling the laboratory fabrication of such prostheses can result in similar long-term results for 3Y-TZP prostheses compared with metal ceramics.

In the posterior region of the mouth adequate reduction for crown preparation is often a challenge and needs to be a balance between required thickness of the planned restoration and preservation of tooth structure. This can be complicated by the need to create adequate axial wall height for resistance and retention form. In 2001, Goodacre and colleagues suggested to maintain a minimum of $4 \mathrm{~mm}$ of occlusal-cervical dimension when preparing molars for full coverage restorations for proper resistance and retention of the prosthesis ${ }^{40}$. Occlusal reduction has been suggested by multiple authors for metal ceramic crowns to be at least $1.5-2 \mathrm{~mm}$ to allow for adequate restorative material thickness $^{40,41}$. An advantage of monolithic zirconia relative to traditional metal ceramic restorations is reduced minimal thickness, due to not requiring space for both a coping and veneering material. Three recently published articles found monolithic zirconia restorations have adequate strength with reduced occlusal thickness. Nakamura et al. showed occlusal thickness has a significant affect on failure of monolithic zirconia crowns during load to fail tests. However, even at a thickness of $0.5 \mathrm{~mm}$ its fracture load was recorded over $5500 \mathrm{~N}^{38}$. Using the same protocol, Nakamura et al. conducted another study evaluating the effect of low temperature degradation and cyclic loading on fail to $\operatorname{load}^{39}$. They found after 100 hours of autoclaving and cyclic loading of 5-300N over 24,000 cycles (simulated 1 year of function) the fail to load value of monolithic zirconia crowns with an occlusal thickness of $0.5 \mathrm{~mm}$ was over $4,000 \mathrm{~N}^{39}$. Another study by Nordahl and colleagues evaluated the load to failure of $0.3 \mathrm{~mm}$ and $0.5 \mathrm{~mm}$ zirconia crowns after thermocycling and 10,000 cycles of $30-300 \mathrm{~N}$ of preload to be $450 \mathrm{~N}$ and $787 \mathrm{~N}$ respectively ${ }^{37}$. These studies suggest monolithic zirconia crowns can withstand 
forces in the molar region and can be utilized in situations where decreased occlusal thickness of the restoration is indicated ${ }^{38}$.

Biocompatibility has been seen as an additional benefit 3Y-TZP has over metal ceramic prosthesis. Although rare, noble and high noble alloys used for fixed restorations have been shown to stimulate hypersensitivity reactions in patients ${ }^{42}$. A report in 2010 investigated replacing metal ceramic FDPs with zirconia prostheses in an attempt to alleviate mucosal irritations related to a metal allergy. Fourteen patients with oral hypersensitivity lesions tested positive in epicutaneous allergy tests for nickel and over half also tested positive to palladium, cobalt and gold ${ }^{25}$. The metal-ceramic FDPs were removed and replaced with zirconia-based restorations and within three weeks, the oral signs and symptoms of allergy resolved. Furthermore, after 3 years in place, no hypersensitivity reactions were observed, supporting the use of 3Y-TZP in patients with metal hypersensitivity ${ }^{25}$.

3Y-TZP based restorations may serve as a suitable alternative to conventional metal ceramics ${ }^{32,43,44}$. Due to the marginal fit and accuracy of CAD/CAM technology, improved esthetics, comparable survival rates, maintenance of strength at reduced occlusal thickness and increased biocompatibility, their numbers could increase and potentially replace the traditional metal ceramics in the future.

\section{Indications for Placement/Replacement of Crowns and FDPs}

The use of full coverage crowns or fixed dental prosthesis is widespread and largely due to identification of a patients need for eliminating existing disease, prevention of future disease, restoration of function, improving appearance, or in posterior endodontically treated teeth to prevent fracture ${ }^{41}$. The indication for a crown or FDP could also be to replace a previously failed restoration. Studies from the United Kingdom have evaluated the prevalence of dental crowns and the frequency of replacement to shed some light on how often such procedures are executed by dental professionals. In 1998 Pine et al. found one-third of the adult population had at least one dental crown ${ }^{45}$. In a 2003 survey of 92 general dentists, it was found within a twelve- 
week period one-third of the crowns fabricated were replacements ${ }^{46}$. The most common cause for the replacements were previous restoration failure, esthetic issues, and recurrent decay $^{46}$. Due to the reported high prevalence in the population and high incident of replacement, the removal of dental prosthesis is a continual problem and expected to continue. Ways of making the procedure efficient and effective are applicable to current daily practice.

The cause for replacement of crowns or fixed dental prosthesis has been evaluated through the years by multiple authors. Schwartz and colleagues in 1970 found the most common cause was due to caries ${ }^{47}$. As a follow-up in 1986, J. E. Walton et al. reported that after a mean time of 8.3 years, caries was still the most common reason for failure $(22 \%)$ followed by porcelain fracture $(16 \%)^{48}$. More recent publications have supported these findings for fixed dental prostheses ${ }^{23,49,50}$. For single crowns, the most common complication has been associated with the need for endodontic therapy, abutment tooth fracture followed by ceramic fracture ${ }^{49,51}$.

Reviews of the literature have also reported the longevity of crowns and FDPs. Scurria et al. in 1998 reported the 10 and 15 year survival rate of fixed dental prosthesis to be $87 \%$ and $69 \%$ respectively ${ }^{52}$. A meta-analysis in 2008 by Pjetursson reported higher survival with an estimated 5-year and 10-year survival of tooth supported FDPs to be $93.8 \%$ and $89.2 \%$ respectively ${ }^{53}$. When comparing survival rates with different materials, similar results were found ${ }^{22,23}$. A systematic review in 2014 found a cumulative 5-year survival of tooth supported and implant supported zirconia crowns to be $95.9 \%$ and $97.1 \%$ respectively ${ }^{22}$. A meta-analysis in 2015 reported the estimated fiveyear survival of zirconia FDPs compared to metal-ceramic FDPs to be $90.4 \%$ and $94.4 \%$ respectively ${ }^{23}$. The lower survival rate for the FDPs was in part due to a higher incidence of veneering ceramic fractures on the zirconia framework. As explained previously, this issue has since been better understood and controlled in the fabrication of such restorations.

Based on the above reports, the replacement of crowns is common due to biologic and technical complications. With comparable expected survivability of metal ceramic 
and zirconia prostheses, the use of such restorations by dental professionals is supported in the short term. In the future it can be expected that a large number of zirconia-based restorations will need to be replaced.

\section{Cutting Efficiency in Dentistry}

Many factors play a role when determining cutting efficiency in dentistry. These include the bur used, the force applied, substrate hardness, handpiece type and coolant flow rate ${ }^{3,454-60}$. All have been shown to affect the cutting efficiency alone or in combination and efforts have been made to determine which combination is most effective. As new technology is available, studies attempt to guide practitioners to the best instruments to use for the clinical situation.

The selection of a dental bur for a procedure is largely due to preference and not science. A survey conducted by Siegel and von Fraunhofer in 1999 reported US dentist favor diamond burs for extracoronal tooth preparation and carbide burs for intracoronal restorations. It was also found that the prosthodontic faculty preferred dental students to use medium grit diamonds for gross tooth reduction and coarser grit diamonds for postgraduate programs $^{61}$. In 2014, Sharma and colleagues recognized a lack of direction

given to practitioners regarding bur selection and conducted a survey of private dentists ${ }^{62}$. A total of 131 general dental practitioners in India responded to the survey and $75 \%$ preferred diamond burs over tungsten carbide or steel for tooth reduction ${ }^{62}$. They also reported a generalized belief that diamond cutting efficiency was a factor of their coarseness $^{62}$. The information from these studies indicates a perception that cutting rates are higher with coarser diamond burs.

In an attempt to test the cutting efficiency as it relates to diamond grit size, Siegel and von Fraunhofer measured the cutting efficiency of three diamond bur grit sizes over three separate cuts into a substitute enamel material. They found no difference between the medium, coarse or super coarse burs during the first cut. When evaluating the total over the three cuts significant differences were seen between the medium and super coarse burs indicating a change in the cutting rate over time ${ }^{54}$. Their work supports the 
conclusion that medium, coarse and super coarse diamond burs have comparable cutting rates over short cutting periods, but when longer cutting times are used coarse and super coarse burs may be more efficient. A recent study verifies this conclusion when using diamond burs on dental zirconia ${ }^{3}$. In 2011, Ohkuma et al. compared diamond burs of two diameters and two particle grit sizes using a constant force to cut $3 \mathrm{Y}_{-}-\mathrm{TZP}^{3}$. They found significant differences when comparing diamond bur particles of $100 \mu \mathrm{m}$ and $200 \mu \mathrm{m}$ as it related to depth of cut and weight of material removed over 120 seconds $^{3}$. They also found a time-dependent decrease in cutting efficiency. The $100 \mu \mathrm{m}$ and $200 \mu \mathrm{m}$ particle burs had a drop in cutting efficiency after 30 and 60 seconds respectively, indicating the larger diamond particle bur maintained its cutting efficiency over a longer period of time $^{3}$. These studies suggest diamond bur particle size has a time-dependent effect on cutting efficiency.

Authors have also demonstrated that cutting efficiency can be increased with an increase in the force applied during cutting on some substrates. Siegel and von Fraunhofer in 1999 evaluated the effect different loads had on the cutting efficiency using diamond burs of varying grit size when cutting an enamel-like substance. They found that the clinical norm of about $100 \mathrm{~g}$ of force was also the optimum pressure to apply for medium grit burs. Interestingly where less pressure resulted in a decreased cutting efficiency, application of more pressure did not produce an increased cutting efficiency with medium grit burs. This is in contrast to coarse grit burs, which did demonstrate an increase cutting efficiency when applying greater force ${ }^{55}$. It should be noted when cutting harder materials such as Y3-TZP, different results were found by Nakamura and colleagues ${ }^{4}$. As the cutting force increased on Y3-TZP, they also noticed an increase in damage to the larger grit diamond burs resulting in decreased cutting efficiency over time ${ }^{4}$. They found that within the first thirty seconds, super coarse diamond burs cut more when a higher force was applied. However, over a five-minute time period, the coarse bur was more efficient and removed more material. They concluded that although larger diamond grit burs showed significantly greater cutting efficiency, higher force may cause more damage to super coarse burs, which may decrease their cutting efficiency over time ${ }^{4}$. 
Two dental handpieces are used in modern dental practice; the electric and airturbine. Ercoli et al. in 2009 noticed a trend toward increasing use of electric handpieces in dental offices and wanted to test both handpieces cutting efficiency. They found the two handpieces performed differently within the first millimeter and second millimeter of cut. The turbine advanced faster with less force in the first millimeter before the cutting instrument was completely engaged into the substrate. They speculated the "free-running speed" of the air turbine might produce greater kinetic energy and power. By contrast, the electric handpiece surpassed the air turbine in the second millimeter of cut when fully immersed in the substrate. This observation was thought to be due to the high consistent torque of the electric handpiece which allows it to run at a constant RPM under load ${ }^{56}$. These findings are supported by Eikenberg, who in 2001 found the efficiency of electric handpiece surpassed the air turbine with increased pressure due to inability for the turbine to have torque control ${ }^{57}$.

In 2010, Choi et al. evaluated the effect of handpiece type and cutting efficiency on different commonly used dental materials. Significant differences were found in cutting efficiency based on handpiece type with some of the materials tested such as high noble metal alloys, silver amalgam and Macor (an enamel-like substance) but not aluminum oxide, base metal alloys, or zirconium oxide ${ }^{58}$. They concluded, "the harder the material, the harder it will be to cut" and the 91 gram force $(.9 \mathrm{~N})$ used in their study may have been insufficient to generate difference in cutting efficiencies between handpieces when cutting hard substrates ${ }^{58}$. Consistent with these findings, Rotella and colleagues found no difference between the use of electric or air turbine hand pieces when evaluating the cutting performance of single use versus multiple use diamond burs $^{59}$. At this time, it appears air turbine and electric handpieces do not differ in cutting efficiency when cutting zirconium oxide at a force of 91 grams.

The last variable shown to affect cutting efficiency is coolant flow rate. The application of a coolant spray during tooth preparations keep temperatures low, removes debris being cut, and keeps the bur clean. In an attempt to evaluate the optimum coolant spray volume, von Fraunhofer and colleagues evaluated five different flow rates with the use of a medium grit diamond bur when cutting an enamel-like substance. They found as 
the flow rate increased, so did the cutting efficiency. They reported the normal coolant spray rate for dentists was $15 \mathrm{ml} / \mathrm{min}$ and increasing it to $25 \mathrm{ml} / \mathrm{min}$ increased the rate of material removal. Interestingly, upon evaluation of the burs, no difference in debris accumulation was found, indicating the effect was not on the bur but on the substrate ${ }^{60}$. Changing the substrate may vary the effect the coolant spray has on cutting efficiency but no evidence to support this is available in the literature.

Based on previous cutting efficiency studies, it appears either electric or air turbine handpieces can be used to cut 3Y-TZP with a force of 91 grams. A coolant water spray of at least $15 \mathrm{ml} / \mathrm{min}$ is advocated and it has been suggested that an increase may also increase the cutting efficiency, however this has not been demonstrated with $3 \mathrm{Y}$ TZP. The selection of diamond bur to use for efficient cutting of 3Y-TZP has not been determined. It has been suggested larger particle size diamond burs are more efficient but those studies only compared two burs and such is insufficient to determine a correlation. Since the development of zirconia removal diamond burs no studies have been conducted to compare those with conventional diamond burs. Lastly, it has been suggested that a diamond bur's cutting efficiency is time-dependent and such timedependent change may also be related to the bur's diamond particle size, however these studies have utilized a stop measure technique which may hide the character of the decrease in cutting efficiency over time or between bur types. At this time it is unclear which diamond bur is most efficient in cutting 3Y-TZP as it relates to diamond particle size and it has not been demonstrated how the rate changes as a dynamic measure over time. 


\section{Chapter III:}

\section{MATERIALS AND METHODS}

\section{Apparatus Construction}

Two custom made experimental devices were built and stabilized on a 10" x 20" plywood base. One device statically held a high-speed air turbine handpiece (W\&H Synea TA-97 LED+, Austria) and was rigidly fixed to the wooden base. The other was a 6 " x 6" wooden platform equipped with an aluminum angle to hold the 3Y-TZP specimen and an extension in the posterior to support the Linear Variable Differential Transformer (LVDT) core. Four aluminum wheels were attached to the underside of the platform, which enabled linear movement along two aluminum tracks rigidly fixed to the wooden base. The LVDT coil assembly was positioned in a cradle attached to the wooden base, allowing the extension on the platform to move within the coil without interference (Figure 1).

The wooden platform and base were treated with polyurethane to reduce dimensional change from water absorption. The aluminum wheels and tracks were treated one time with a petroleum-based lubricant (WD-40, San Diego, CA) immediately after fabrication to decrease friction. 


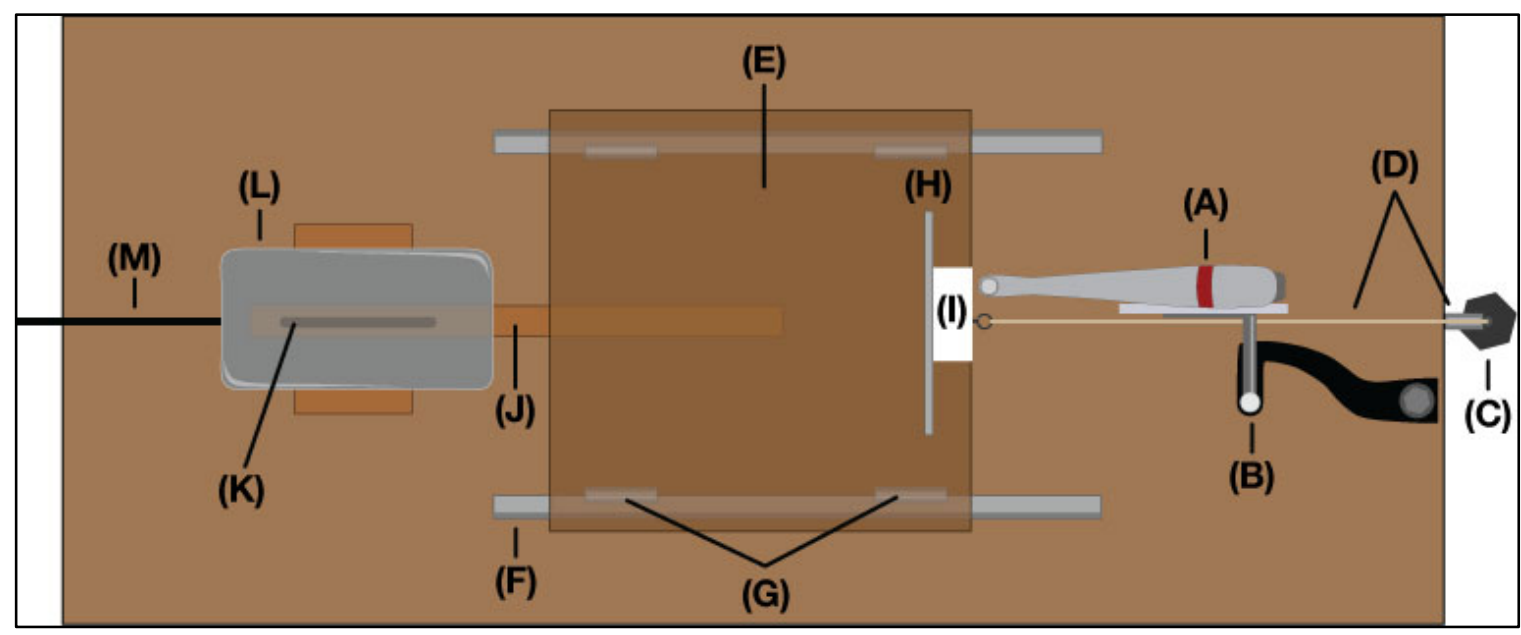

Figure 1. Experiment Apparatus.

(A) Handpiece, (B) Handpiece Stand, (C) Weights, (D) Pulley and Line, (E) Platform, (F) Aluminum Track, (G) Aluminum wheels, (H) Aluminum angle, (I) YSZ block, (J) Support for core, (K) LVDT Core, (L) LVDT Coil Assembly, (M) Leads to Signal Conditioner (Illustrated by Ryan Alexander).

\section{Data Recording Instrumentation and Configuration}

A Lucas Schevitz 503XS-A LVDT with an ATA-2001 Signal Conditioner (Measurement Specialties, Hampton, VA) was used to measure the linear displacement of the platform with an output in voltage. The signal conditioner was calibrated with the LVDT according to the manufacturer's instructions for unipolar voltage output and operated at the manufacturer's default settings of $3.5 \mathrm{~V}$ at $2.5 \mathrm{kHz}$. The LVDT stroke is 1 inch or 10 volts and the voltage output can be converted to distance as 1 volt $=0.1$ inch $=2.54 \mathrm{~mm}$.

A Personal Daq/3000 series data acquisition device (Measurement Computing, Norton, MA) received the real-time output generated by the LVDT conditioner and plotted the measured voltage over time according to custom programmed specifications in the Daq software. Set-up of data recording instrumentation and apparatus can be seen in Figure 2. 


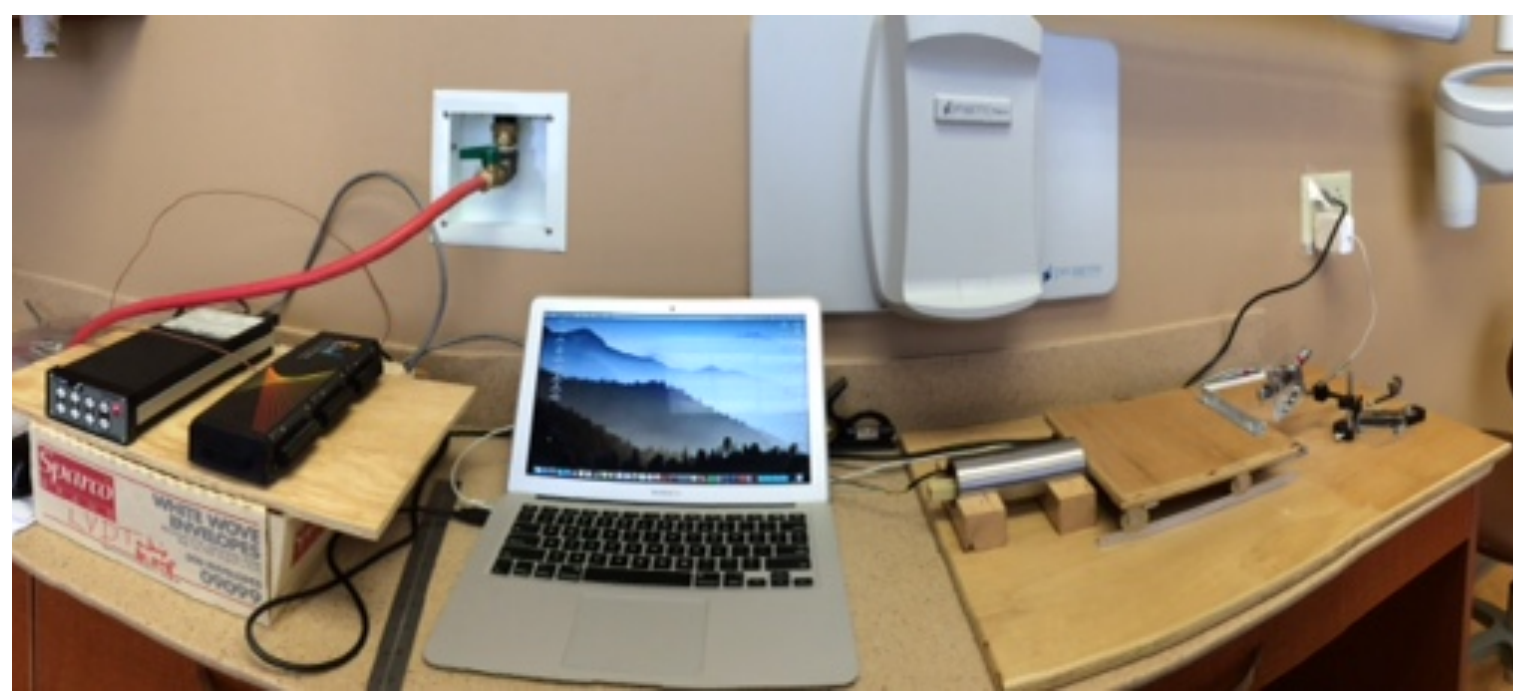

Figure 2. Experiment Assembly

From left to right: LVDT signal conditioner, Personal Daq/3000, laptop used to run Daq/3000 software, experiment apparatus.

A Draper dial caliper (Hampshire, UK) with graduations of $0.02 \mathrm{~mm}$ was used to measure the depth of each cut and recorded in the nearest tenth of a millimeter.

\section{Materials}

Three rectangular IPS e.max ZirCAD B40L blocks with a dimension of $39 \times 19 \times 15.4 \mathrm{~mm}$ were sintered according to the manufacturer's instructions (Ivoclar Vivadent, Buffalo, NY). Seven diamond burs were selected from two companies based on diamond particle size and dimension of the bur shank (Table 1). All burs were reported to be $1.4 \mathrm{~mm}$ in diameter, parallel sided and chamfer ended. Ten replicates of each bur type were used to equal 70 total test burs. 
Table 1. Bur Characteristics.

\begin{tabular}{l|l|c|c}
\hline $\begin{array}{c}\text { Letter } \\
\text { assignment }\end{array}$ & Manufacturer & Name & Diamond grit size \\
\hline A & Brasseler & KS2 & Super Coarse $(150 \mu \mathrm{m})$ \\
$\mathrm{B}$ & Brasseler & 8881 & Fine $(30 \mu \mathrm{m})$ \\
$\mathrm{C}$ & Brasseler & BRKS2 & Coarse $(125 \mu \mathrm{m})$ \\
$\mathrm{D}$ & Komet & $4 \mathrm{ZR}^{\mathrm{a}}$ & - \\
$\mathrm{E}$ & Komet & 6881 & Coarse $(151 \mu \mathrm{m})$ \\
$\mathrm{F}$ & Komet & 881 & Medium $(107 \mu \mathrm{m})$ \\
$\mathrm{G}$ & Komet & 2979 & Super Coarse $(181 \mu \mathrm{m})$ \\
\hline
\end{tabular}

${ }^{a}$ Recommended by Komet for cutting 3Y-TZP, no information provided regarding diamond particle size.

\section{Protocol}

The sequence of bur testing was determined by block randomization. Before the start of each run the bur was paralleled to the 3 Y-TZP block, adjusted to a $1.5 \mathrm{~mm}$ depth, and the $3 \mathrm{Y}-\mathrm{TZP}$ on the platform was positioned to be in contact with the bur. A $0.9 \mathrm{~N}$ force was then exerted on the $3 \mathrm{Y}-\mathrm{TZP}$ in the direction of the bur by vertically suspending 91 grams of fishing weights over a pulley at the end of the wooden base. An additional 1.25 grams of weight was added to overcome breakaway friction of the platform.

The Daq was initiated and data recording occurred for a two second count before pressure was exerted on the rheostat. The two-second delay allowed a recording of a zero position of the LVDT. Maximal pressure was exerted on the rheostat with a measured coolant spray rate of $16 \mathrm{~mL} / \mathrm{min}$ for a continuous 5 minutes. At the end of each fiveminute test interval, the data file was saved, the depth of the slot created in the $3 \mathrm{Y}$-TZP was recorded with a dial caliper, the next bur in series was placed, the zirconia block was adjusted to expose an uncut surface and the process was repeated. 


\section{Statistical Analysis}

The data consists of recorded voltages measured from time zero to 5 minutes with measurements taken every tenth of a second for ten replicates of seven bur types. Average voltages were computed for each bur per second. The slope of the best fit lines for each bur replicate was computed for 4 time intervals. The slope of the line was used for comparison between and within test variables and can be interpreted as the steeper the slope then the greater the cutting efficiency. Time was divided into periods: Period 0 (02 seconds), Period 1 (2-100 seconds), Period 2 (100-200 seconds), and Period 3 (200-300 seconds). Period 0 reflects a two second lapse before cutting to verify a true start position with the LVDT, thus will not be included in analysis. The depth of cut for each bur was used as a confounder.

The slopes of the lines were compared using a mixed-effects ANOVA to define the variables' effect on cutting efficiency. The variables showing significance were further evaluated using a post-hoc Tukey-Kramer Honestly Significant Difference (TukeyKramer HSD) or Fisher Least Significant Difference (LSD) to assess differences between pairs of means. The statistic software used to evaluate the data was JMP® Pro Version 12 (Cary, NC). 


\section{Chapter IV}

\section{RESULTS}

Averaged distance each bur traveled can be seen in Figure 3 as a function of time depicting the overall trends in the cutting efficiency. Mean distance cut by each bur over 5 minutes can be seen in Figure 4. Statistically significant effects on cutting efficiency were seen among the seven diamond burs, the periods, and depths in cutting the $3 \mathrm{Y}-\mathrm{TZP}$. When evaluating the interaction between the effects of burs and periods on cutting efficiency, no significant difference was found (Table 2).

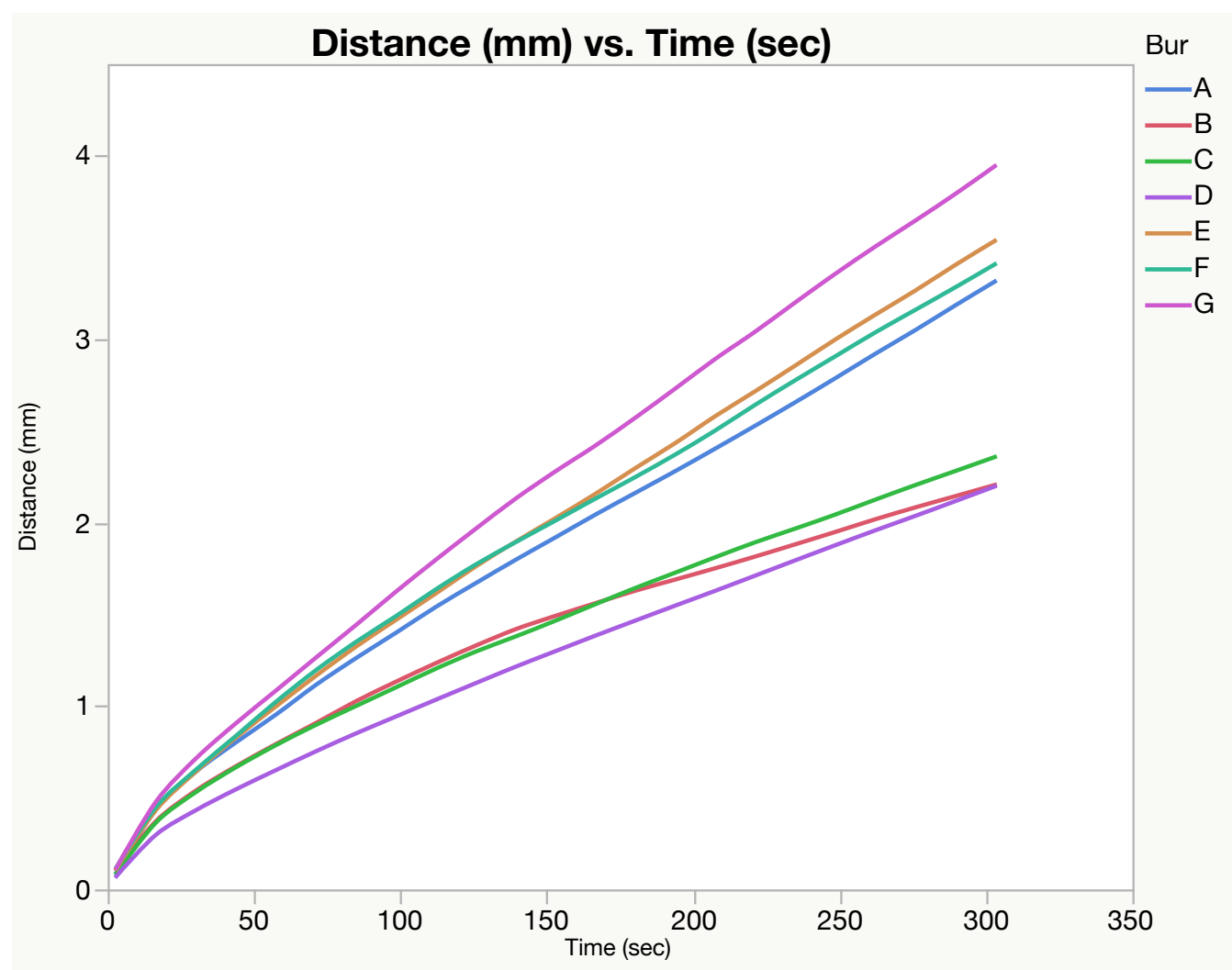

Figure 3. Cutting efficiency of test burs over 5 minutes (JMP/Pro Ver12, SAS Inst. Inc., Cary, NC). 


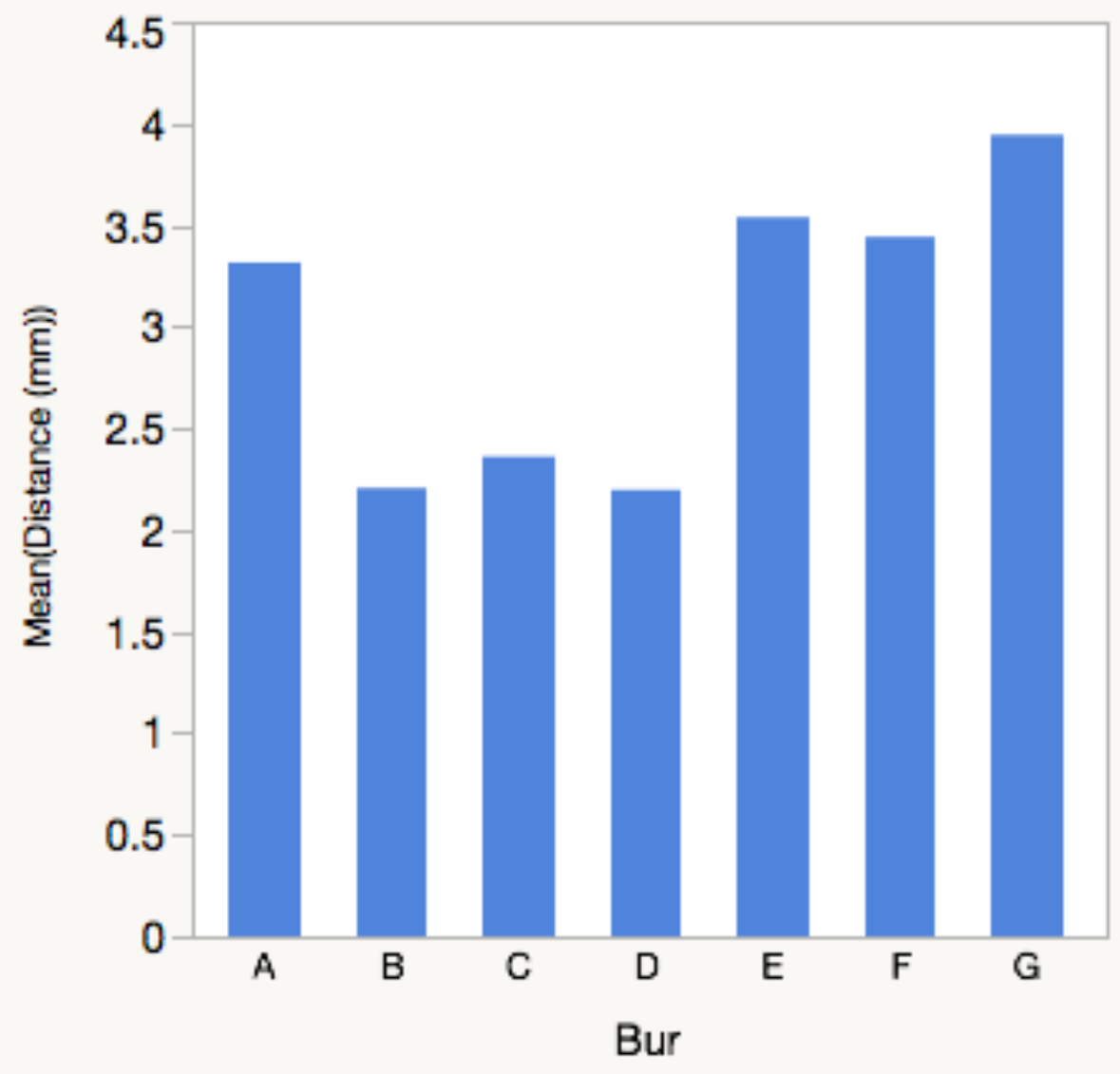

Figure 4. Mean distance cut of each bur group after 5 minutes (JMP/Pro Ver12, SAS Inst. Inc., Cary, NC).

Table 2. Mixed Effects ANOVA.

\begin{tabular}{|l|r|r|r|r|}
\hline Variables & DF & DFDen & F Ratio & Prob $>$ F \\
\hline Bur & 6 & 62 & 4.8157 & $0.0004^{\mathrm{a}}$ \\
\hline Period & 2 & 126 & 89.4878 & $<.0001^{\mathrm{a}}$ \\
\hline Period*Bur & 12 & 126 & 0.8813 & 0.5676 \\
\hline
\end{tabular}

${ }^{a}$ Represents significant differences at the $\mathrm{P}<0.05$ interval (JMP/Pro Ver12, SAS Inst. Inc., Cary, NC). 
To define the significance between the seven diamond burs, a Tukey-Kramer HSD was completed (Table 3). In descending order bur types G (2979/super coarse/181 $\mu \mathrm{m})$, E

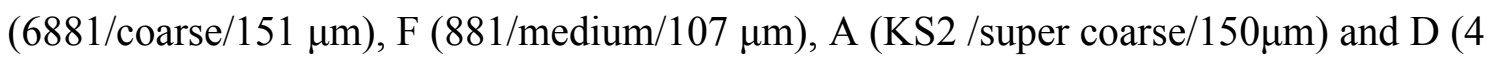
ZR) were significantly more efficient in cutting the $3 \mathrm{Y}$-TZP in 5 minutes compared to bur types C (BRKS2/coarse/125 $\mu \mathrm{m})$ and B (8881/fine/30 $\mu \mathrm{m})$.

Table 3. Tukey-Kramer HSD defining significance between burs.

\begin{tabular}{|llllr|}
\hline Bur & & & & $\begin{array}{r}\text { Least Sq Mean Rate } \\
(\mathbf{m m} / \mathbf{s e c})\end{array}$ \\
\hline G & A & & & 0.0118 \\
\hline E & A & B & 0.0112 \\
\hline F & A & B & & 0.0109 \\
\hline A & A & B & C & 0.0102 \\
\hline D & A & B & C & 0.0078 \\
\hline C & & B & C & 0.0069 \\
\hline B & & & C & 0.0062 \\
\hline
\end{tabular}

Burs not connected by the same letter showed significant differences (JMP/Pro Ver12, SAS Inst. Inc., Cary, NC).

Significant differences were found between time periods. A Tukey-Kramer HSD further defined the significance. All burs were significantly more efficient in cutting $3 \mathrm{Y}$ TZP in Period 1 compared to Periods 2 and 3 (Table 4).

Table 4. Tukey-Kramer HSD defining significance between periods.

\begin{tabular}{|c|c|c|}
\hline Period & & $\begin{array}{r}\text { Least Sq Mean Rate } \\
(\mathrm{mm} / \mathrm{sec})\end{array}$ \\
\hline 1 & A & 0.0116 \\
\hline 2 & B & 0.0082 \\
\hline 3 & B & 0.0081 \\
\hline
\end{tabular}

Periods not connected by the same letter show significant differences (JMP/Pro Ver12, SAS Inst. Inc., Cary, NC). 
The depth of cut for each bur was used as a confounder and a Tukey-Kramer HSD was completed to evaluate the differences between bur types. No significant difference was found between bur types when averaging the depths for each bur type.

Comparison of burs and periods simultaneously did not result in significant differences; meaning no significant difference was found when comparing bur-to-bur change between periods (Figure 5).

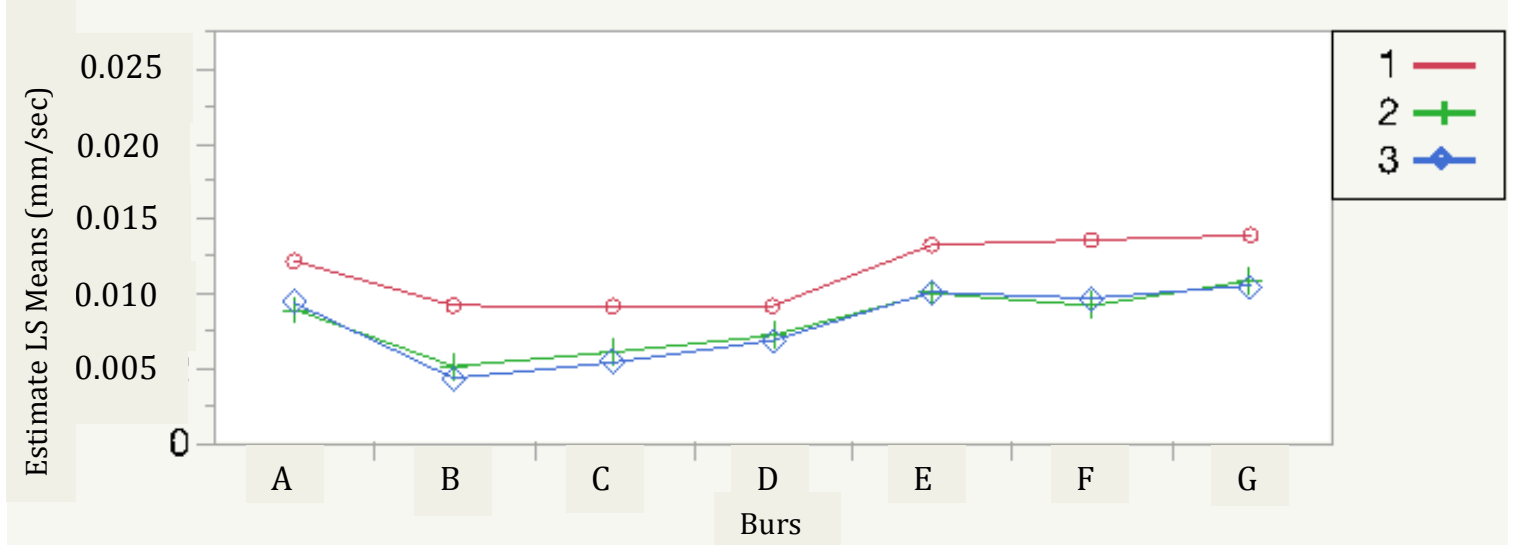

Figure 5. Cutting efficiency average for bur groups in each Period (JMP/Pro Ver12, SAS Inst. Inc., Cary, NC).

The averaged distance for each bur in Period 1 can be seen in Figure 6. A oneway ANOVA was used to evaluate the significance which was found in Period 1 as it relates to cutting efficiency (Figure 7). Significant differences between burs were found at a $\mathrm{p}=0.0162$ (Figure 7). The post-hoc Fisher LSD, as seen in Table 5, defined the significance. The four burs displaying a significantly greater cutting efficiency in Period 1 in descending order were G (2979/super coarse/181 $\mu \mathrm{m}), \mathrm{F}$ (881/medium/107 $\mu \mathrm{m}), \mathrm{E}$

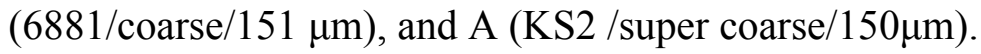




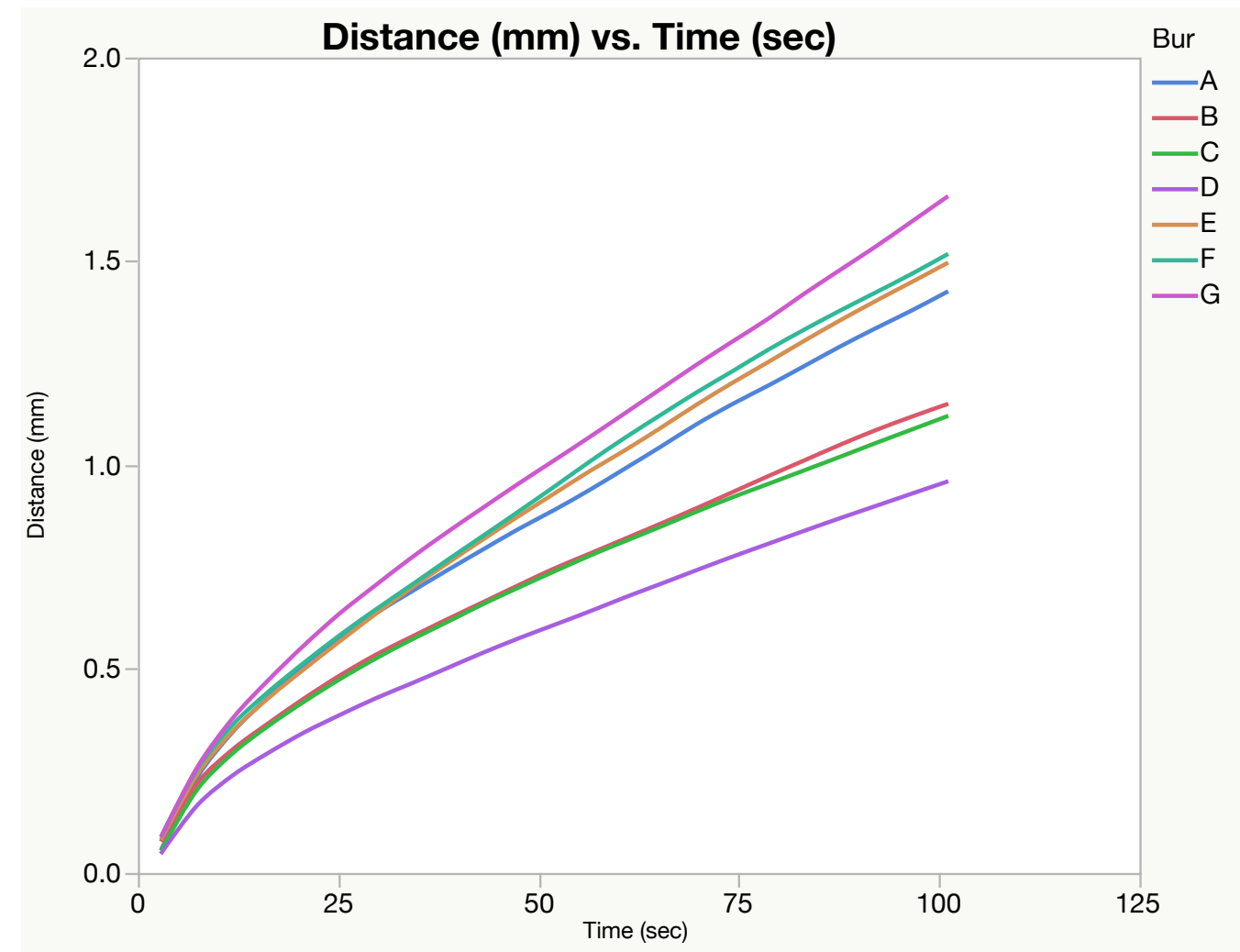

Figure 6. Cutting efficiency of test burs in Period 1(JMP/Pro Ver12, SAS Inst. Inc., Cary, NC). 


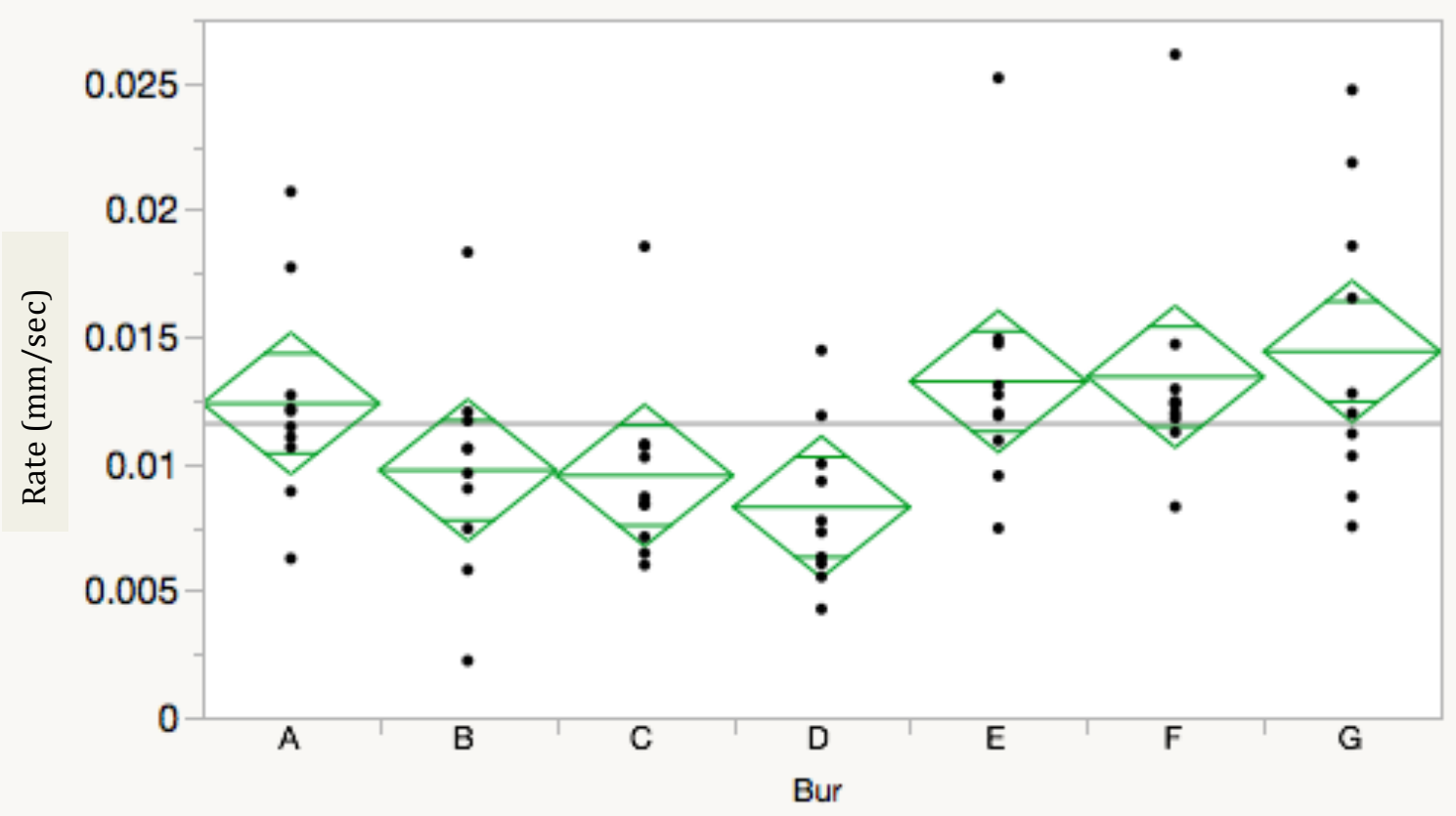

Figure 7. Oneway ANOVA depicting cutting efficiency in Period 1(JMP/Pro Ver12, SAS Inst. Inc., Cary, NC).

Table 5. Fisher LSD defining differences between burs in Period 1.

\begin{tabular}{|lccrr|}
\hline Bur & & & & Mean Rate $(\mathrm{mm} / \mathbf{s e c})$ \\
\hline G & A & & & 0.0144 \\
\hline F & A & B & & 0.0134 \\
\hline E & A & B & & 0.0132 \\
\hline A & A & B & & 0.0124 \\
\hline B & & B & C & 0.0097 \\
\hline C & & B & C & 0.0095 \\
\hline D & & & C & 0.0083 \\
\hline
\end{tabular}

Burs not connected by the same letter show significant differences (JMP/Pro Ver12, SAS Inst. Inc., Cary, NC). 


\section{Chapter V}

\section{DISCUSSION}

The present study investigated the cutting efficiency of diamond burs on sintered 3Y-TZP. Based on the results the null hypothesis that bur type will not affect cutting efficiency was rejected. However, the null hypothesis that the type of bur would not affect the time at which bur cutting efficiency declined during the five-minute observation was accepted. All burs had a decline after the first 100 seconds, but the burto-bur decline comparison was not significantly different.

The top two performers with regards to cutting efficiency over 5 minutes were the 2979 (bur G) and 6881 (bur E), which also have the largest reported diamond grit size of the burs sampled as $181 \mu \mathrm{m}$ and $151 \mu \mathrm{m}$ respectively. However, when evaluating the degree of efficiency of each bur to cut 3Y-TZP with regards to manufacturer reported grit size, inconsistencies were noticed in the data. In descending order burs G (2979/super coarse/181 $\mu \mathrm{m})$, E $(6881 /$ coarse/151 $\mu \mathrm{m}), \mathrm{F}(881 /$ medium/107 $\mu \mathrm{m}), \mathrm{A}(\mathrm{KS} 2 /$ super coarse $/ 150 \mu \mathrm{m})$ and D (4 ZR) were found to have significantly greater cutting efficiency in comparison to burs $\mathrm{C}(\mathrm{BRKS} 2 /$ coarse $/ 125 \mu \mathrm{m})$ and B $(8881 /$ fine $/ 30 \mu \mathrm{m})$. An interesting finding is bur F (881) is reported to be medium grit with diamond particles of $107 \mu \mathrm{m}$ and $\mathrm{C}(\mathrm{BRKS} 2)$ is a coarse grit diamond with particle size of $125 \mu \mathrm{m}$. If particle size alone was the determining factor for cutting efficiency bur $\mathrm{H}$ should not have outperformed bur C. One possible cause for the discrepancy is the design of bur C. The manufacturer promotes this bur to cut enamel more efficiently by incorporating free space between the diamond particles to reduce bur clogging with debris ${ }^{63}$. Problems may arise when cutting harder surfaces such as 3Y-TZP. The determining factor for cutting efficiency may be more dependent on diamond surface area available to contact the substrate rather than space for debris to escape.

When evaluating the decline of cutting efficiency over time, no significant difference was noted in a bur-to-bur comparison. All burs seem to decline at a similar rate after the first 100 seconds of use/Period 1 (Figure 5). In addition, the performance in 
the subsequent time periods appears to be similar and follow a linear pattern. These results suggest a significant decline in bur cutting efficiency occurs after the first 100 seconds, which then stabilizes for the remaining 5-minute observation. In contrast, a study by Nakamura in 2015 reported observing a linear pattern of cutting through the first 5 minutes when using coarse and super coarse diamonds on 3Y-TZP. They reported a decline in efficiency only during the evaluation from 5 to 10 minutes of cutting. A possible cause for the difference may be due to the differences in data collection. In the present study the data was collected continually as a dynamic process. The other study stopped at each 30-second time interval to collect measurements. Cause of error in that study could be due to inconsistent data collection between and among samples in addition to not being able to visualize trends that may be occurring within the 30-second time interval. Their data reflects a start and a stop point, leaving out all the information regarding the nature of the cutting in-between those data points. Although the time when the burs decreased efficiency was not consistent between studies, the cause for the decrease may be the same. A SEM evaluation of the diamond burs used by Nakamura et al. revealed damage to the diamond burs including chipping, pull out and wear of the diamond particles and was indicated as the cause for the decrease in cutting efficiency ${ }^{4}$. In an attempt to investigate the differences between the studies, future evaluations could expand the cutting time beyond 5 minutes to determine if and when a second significant difference in cutting efficiency occurs.

The error introduced by the inability to control for bur depth seems to be evenly distributed among the burs. Reporting the data as a mean for each bur reduces the variability and allows a summative evaluation of bur-to-bur comparison. Although significant differences were not found between the mean depths of each bur, Figure 2 shows a trend toward bur D (4 ZR) used at a greater depth. This may have caused bur D to be less efficient in the given results than if the depth had been more accurately controlled. Based on the results in Table 3, bur D's cutting efficiency represented a "middle-of-the-road" performance compared to the other burs. Based on evaluations by Nakamura et al., diamond bur cutting efficiency is significantly affected by the cutting depth of zirconia, thus the results in the present study may underestimate bur D's actual 
cutting efficiency when compared to the other burs ${ }^{4}$. The 4 ZR, "fo(u)r zirconia", is marketed as a zirconium oxide crown remover by having characteristics of long serviceability, superior zirconia substance removal, and improved adhesion of the diamond grains ${ }^{64}$. The question remains, if the depth was controlled more accurately would this zirconia cutting bur out performed the rest of the burs in this study?

The results of this study show bur G, having the largest diamond particle size of the seven burs, to be the most efficient cutting of 3Y-TZP in both Period 1 and across all Periods. These results support the work of others in relation to diamond grit size and cutting efficiency of 3Y-TZP. Two other studies found larger grit diamond burs to be capable of greater cutting depths over time compared to smaller grit diamond burs when comparing particle sizes of $100 \mu \mathrm{m}$ and $200 \mu \mathrm{m}$ in one study and $187 \mu \mathrm{m}$ and $305 \mu \mathrm{m}$ in the other $^{3,4}$. In contrast, an article by Blue et al. failed to find a significant difference in diamond bur particle size and zirconia substrate removal when evaluating which bur is most effective when preparing 3Y-TZP implant abutments ${ }^{65}$. The burs tested had particle sizes $150 \mu \mathrm{m}(\mathrm{KS} 2), 100 \mu \mathrm{m}(881)$ and $30 \mu \mathrm{m}(8881)$, were used in air turbine handpieces, at a force of $0.9 \mathrm{~N}$, and with a cutting time of 34 seconds $^{65}$. A possible cause for their conflicting results could be due to the particle size of the burs they used and the limited application time. The KS2, 881 and 8881 burs were also used in the present study with reference names of burs A, F, and B respectively. As seen in Table 5, this study had similar results of no significant difference between those burs when evaluating bur-to-bur comparisons within Period 1. If they included burs with larger particle size such as bur G, with particle size of $181 \mu \mathrm{m}$ or the super coarse used by Nakamura et al. of $305 \mu \mathrm{m}$ the results of their study may have produced significant differences between burs ${ }^{4}$. Furthermore, they only allowed the cutting time to be 34 seconds and measured efficiency by material removal by change in weight. Given the material is very difficult to cut and the amount of measurable material lost is minute, the validity of their results are strictly dependent on the accuracy of the measuring device and meticulous cleaning and drying the samples before each weighing. Additionally to complicate the validity of their results, as described by Siegel and von Fraunhofer in 2000, cutting efficiency is dependent on diamond particle size and duration of use ${ }^{54}$. Their work supports that 
medium, coarse and super coarse diamond burs have comparable cutting rates over short cutting periods but when longer cutting times are used coarse and super coarse burs may be more efficient ${ }^{54}$. Despite Blue et al.'s findings being inconsistent with those of the present study, aspects of the experimental design may be the cause for the inability to define significance in their results.

An advantage of this study design compared to previous studies of cutting efficiency is the dynamic recording of cutting depth over time. The utilization of the LVDT and Daq allowed real-time data collection as the diamond bur advanced through the $3 \mathrm{Y}$-TZP specimens and may provide fewer inconsistencies throughout the data collection. Most studies of cutting efficiency use weight or calipers to determine material lost or depth of cut, which requires planned stopping points to provide opportunities to collect data over time. The current design allowed a more consistent data collection method across samples and eliminated multiple stopping points necessary, which could act as a source of error.

One disadvantage of this study was not exposing the $3 \mathrm{Y}$-TZP blocks to conditions similar to the oral environment. Multiple authors have investigated the detrimental effect the oral environment can have on the transformation toughening nature of $3 \mathrm{Y}-\mathrm{TZP} \mathrm{P}^{66-71}$. This is the result of low-temperature degradation (LTD) and cyclic loading which can decrease the fracture resistance of zirconia by causing spontaneous phase transformations at the surface. In a meta-analysis of the literature, Pereira et al. evaluated LTD and the effect time, temperature, pressure and m-phase content have on the decrease in flexural strength. They found the greatest resultant decrease in flexural strength with LTD occurs when the time of LTD is increased to or beyond 20 hours and the quantity of monoclinic phase reaches $50 \%$ or more ${ }^{70}$. They reported it results in a decrease in the flexural strength of $3 \mathrm{Y}$-TZP by $231 \mathrm{~N}$ and $212 \mathrm{~N}$ respectively ${ }^{70}$. Kvam and Karlsson in 2013 compared the flexural strength of 3 different zirconia materials after LTD for one week in hot $4 \%$ volume acetic acid and found a decrease of $100-200 \mathrm{MPa}$ in a load to fail evaluation. Even if or when this decrease occurs, Nakamura et al. determined it still allowed zirconia molar crowns to have sufficient strength ${ }^{39}$. The effect however has not been evaluated as it regards to cutting efficiency and removal of zirconia prosthesis and 
could be investigated in future studies to accurately simulate changes which may occur to $3 \mathrm{Y}-\mathrm{YZP}$ in the oral environment.

Another disadvantage of this study was the lack of surface manipulations applied to the $3 \mathrm{Y}-\mathrm{TZP}$. During prosthesis fabrication and delivery it is common for the laboratory to sandblast the inner surface for cementing purposes and/or grinding to occur chair-side during the fitting of the prosthesis in the patient's mouth. When stress is applied to the surface of zirconia, phase transformations can occur. The affect those transformations have on the mechanical properties depends on the amount of transformations that occur and the depth they extend. Manipulations on 3Y-TZP can stimulate the formation of a compressive surface layer which can increase the strength of the zirconia ${ }^{18}$. However, aggressive grinding can introduce deep surface flaws that extend beyond the compressive surface layer. The deep flaws act as stress concentrators and decrease the strength ${ }^{18}$. Kosmac and colleagues demonstrated this phenomenon when evaluating sandblasting and surface grinding effects on 3Y-TZP. Sandblasting increased the strength of 3Y-TZP by formation of a compressive layer but surface grinding did not. The authors attributed these findings to heat generation and the more aggressive nature of grinding, which may produce deeper flaws extending beyond the formed compressive layer ${ }^{72}$. Application of sandblasting and surface grinding to test specimens may assist in replicating possible changes to the mechanical properties of $3 \mathrm{Y}$ TZP that occur during prosthesis fabrication and clinical delivery.

In order to determine which bur will maximize cutting efficiency, one has to evaluate not only the bur(s) in question but also the technique employed and the goal to be accomplished. As demonstrated by Nakamura et al., when using a super coarse diamond bur and doubling the force, the initial cutting efficiency can be greatly increased but at the expense of bur durability ${ }^{4}$. At the end of the 5-minute test run using the higher force, the smaller diamond particle bur removed more material, thus demonstrating particle size is not the only factor when evaluating cutting efficiency. Additionally the goal is an important factor in choosing diamond bur cutting instruments. For example a study by Ohkuma et al. recognized not only the need to remove bulk 3Y-TZP material to cut off failing restorations but also for gross reductions in the laboratory during 
fabrication ${ }^{3}$. They evaluated two diameters of $100 \mu \mathrm{m}$ and $200 \mu \mathrm{m}$ diamond particle burs to evaluate distance cut into $3 \mathrm{Y}-\mathrm{TZp}$ substrate and mass of material removed. They found that the narrow diameter $200 \mu \mathrm{m}$ bur was most efficient to cut a larger distance into the zirconia and the larger diameter $200 \mu \mathrm{m}$ bur produced a greater mass reduction in the 3Y-TZP sample ${ }^{3}$. Based on their results removing a failing prosthesis by cutting multiple slots would be best executed by using a smaller diameter large particle diamond bur.

An alternative technique to remove a non-serviceable zirconia crown has been proposed by multiple authors ${ }^{73,74}$. A case study report in a non peer-reviewed article by J. Cranska examined the ability to remove ceramic restorations with the application of a hard tissue Er:YAG dental laser. Dr. Cranska demonstrated the removal of monolithic or bilayered zirconia crowns by applying laser light energy slowly over all surfaces of the crowns. He reports that within two minutes he can remove the prosthesis with a dental instrument and the internal surface of the restorations are cement free ${ }^{73}$. Rechmann et al. in 2014 conducted a laboratory proof-of-principle study to evaluate the ability to debond lithium disilicate and zirconia crowns without damaging the underlying tooth structure ${ }^{74}$. They found zirconia requires significantly more laser energy to debond than lithium disilicate due to zirconia transmitting $80 \%$ less Er:YAG laser energy to the cement layer. They also reported the average time for removal was 312 seconds, and no damage was noted to the underlying tooth structure. They concluded it is possible to remove zirconia crowns with an Er:YAG, however additional studies evaluating the effect of the high level laser energy need to be conducted before advocating it for clinical use ${ }^{74}$. However, even if studies are published verifying the safe use of laser energy to remove 3Y-TZP crowns, this technique will be limited to those practitioners who have access to such technology in their practice. 


\section{Chapter VI:}

\section{SUMMARY}

The use of 3Y-ZTP as a dental restorative material is increasing due to its favorable mechanical properties. Unfortunately dental technology has not advanced to the point of preventing replacement of previously placed restorations. This study attempted to provide information on the best diamond bur to use for removal of a zirconia restoration via sectioning. This study only provides data for one type of 3Y-TZP and seven burs from two manufacturers. The data does give guidance to practitioners for which bur(s) are most efficient when cutting dental zirconia in attempt to limit wasted time and resources for the dental practitioner and the patient.

\section{CONCLUSIONS}

1) Super coarse, coarse and medium diamond particle burs are more efficient in cutting 3Y-TZP than fine diamond burs.

2) Bur cutting efficiency is maximized for all burs when limiting the cutting time of 3Y-TZP to 100 seconds with a new diamond bur.

3) Super coarse diamond particle burs are the most efficient in cutting 3Y-TZP throughout the 5-minute testing time. 


\section{REFERENCES}

1. Materials for fixed prosthodontics. In: Wiens JP, ed. Fundamentals of occlusion. American College of Prosthodontists; 2015:583.

2. Christensen GJ. Removing zirconia or lithium disilicate crowns. Dental Economics. 2014;104(7):January 25, 2016.

3. Ohkuma K, Kazama M, Ogura H. The grinding efficiency by diamond points developed for yttria partially stabilized zirconia. Dent Mater J. 2011;30(4):511-516. doi: JST.JSTAGE/dmj/2010-152 [pii].

4. Nakamura K, Katsuda Y, Ankyu S, et al. Cutting efficiency of diamond burs operated with electric high-speed dental handpiece on zirconia. Eur J Oral Sci. 2015. doi: 10.1111/eos.12211 [doi].

5. The glossary of prosthodontic terms. J Prosthet Dent. 2005;94(1):10-92.

6. Anusavice KJ. Phillips' science of dental materials. Eleventh ed. St. Louis, Missouri: Saunders; 2003.

7. Manicone PF, Rossi Iommetti P, Raffaelli L. An overview of zirconia ceramics: Basic properties and clinical applications. J Dent. 2007;35(11):819-826. doi: S03005712(07)00141-8 [pii].

8. Piconi C, Maccauro G. Zirconia as a ceramic biomaterial. Biomaterials. 1999;20(1):125. doi: S0142-9612(98)00010-6 [pii].

9. Denry I, Kelly JR. State of the art of zirconia for dental applications. Dent Mater. 2008;24(3):299-307. doi: S0109-5641(07)00113-3 [pii].

10. Conrad HJ, Seong WJ, Pesun IJ. Current ceramic materials and systems with clinical recommendations: A systematic review. J Prosthet Dent. 2007;98(5):389-404. doi: S0022-3913(07)60124-3 [pii].

11. Hisbergues M, Vendeville S, Vendeville P. Zirconia: Established facts and perspectives for a biomaterial in dental implantology. J Biomed Mater Res B Appl Biomater. 2009;88(2):519-529. doi: 10.1002/jbm.b.31147 [doi].

12. Raigrodski AJ. Contemporary materials and technologies for all-ceramic fixed partial dentures: A review of the literature. J Prosthet Dent. 2004;92(6):557-562. doi: S0022391304006158 [pii].

13. Guazzato M, Albakry M, Ringer SP, Swain MV. Strength, fracture toughness and microstructure of a selection of all-ceramic materials. part II. zirconia-based dental 
ceramics. Dent Mater. 2004;20(5):449-456. doi: 10.1016/j.dental.2003.05.002 [doi].

14. Christel P, Meunier A, Heller M, Torre JP, Peille CN. Mechanical properties and short-term in-vivo evaluation of yttrium-oxide-partially-stabilized zirconia. J Biomed Mater Res. 1989;23(1):45-61. doi: 10.1002/jbm.820230105 [doi].

15. Denry I, Kelly JR. Emerging ceramic-based materials for dentistry. J Dent Res. 2014;93(12):1235-1242. doi: 10.1177/0022034514553627 [doi].

16. Miyazaki T, Nakamura T, Matsumura H, Ban S, Kobayashi T. Current status of zirconia restoration. Journal of Prosthodontic Research. 2013;57(4):236 <last_page> 261. doi: 10.1016/j.jpor.2013.09.001.

17. Komine F, Blatz MB, Matsumura H. Current status of zirconia-based fixed restorations. J Oral Sci. 2010;52(4):531-539. doi: JST.JSTAGE/josnusd/52.531 [pii].

18. Luthardt RG, Holzhuter MS, Rudolph H, Herold V, Walter MH. CAD/CAMmachining effects on Y-TZP zirconia. Dent Mater. 2004;20(7):655-662. doi: 10.1016/j.dental.2003.08.007 [doi].

19. Christensen GJ. Is the rush to all-ceramic crowns justified? J Am Dent Assoc. 2014;145(2):192-194. doi: 10.14219/jada.2013.19 [doi].

20. Guncu MB, Cakan U, Muhtarogullari M, Canay S. Zirconia-based crowns up to 5 years in function: A retrospective clinical study and evaluation of prosthetic restorations and failures. Int J Prosthodont. 2015;28(2):152-157. doi: 10.11607/ijp.4168 [doi].

21. Håff A, Löf H, Gunne J, Sjögren G. A retrospective evaluation of zirconia-fixed partial dentures in general practices: An up to 13-year study. Dental Materials. 2015;31(2):162 <last_page> 170. doi: 10.1016/j.dental.2014.11.009.

22. Larsson C, Wennerberg A. The clinical success of zirconia-based crowns: A systematic review. Int J Prosthodont. 2014;27(1):33-43. doi: 10.11607/ijp.3647 [doi].

23. Pjetursson BE, Sailer I, Makarov NA, Zwahlen M, Thoma DS. All-ceramic or metalceramic tooth-supported fixed dental prostheses (FDPs)? A systematic review of the survival and complication rates. part II: Multiple-unit FDPs. Dent Mater. 2015;31(6):624-639. doi: 10.1016/j.dental.2015.02.013 [doi].

24. Gonzalo E, Suarez MJ, Serrano B, Lozano JF. A comparison of the marginal vertical discrepancies of zirconium and metal ceramic posterior fixed dental prostheses before and after cementation. J Prosthet Dent. 2009;102(6):378-384. doi: 10.1016/S0022-3913(09)60198-0 [doi]. 
25. Gokcen-Rohlig B, Saruhanoglu A, Cifter ED, Evlioglu G. Applicability of zirconia dental prostheses for metal allergy patients. Int J Prosthodont. 2010;23(6):562-565.

26. Ortega R, Gonzalo E, Gomez-Polo M, Suarez MJ. Marginal and internal discrepancies of posterior zirconia-based crowns fabricated with three different CAD/CAM systems versus metal-ceramic. Int J Prosthodont. 2015;28(5):509-511. doi: $10.11607 / i j p .4359$ [doi].

27. Reich S, Wichmann M, Nkenke E, Proeschel P. Clinical fit of all-ceramic three-unit fixed partial dentures, generated with three different CAD/CAM systems. Eur J Oral Sci. 2005;113(2):174-179. doi: EOS197 [pii].

28. Rinke S, Kramer K, Burgers R, Roediger M. A practice-based clinical evaluation of the survival and success of metal-ceramic and zirconia molar crowns: 5-year results. J Oral Rehabil. 2015. doi: 10.1111/joor.12348 [doi].

29. Vigolo P, Fonzi F. An in vitro evaluation of fit of zirconium-oxide-based ceramic four-unit fixed partial dentures, generated with three different CAD/CAM systems, before and after porcelain firing cycles and after glaze cycles. $J$ Prosthodont. 2008;17(8):621-626. doi: 10.1111/j.1532-849X.2008.00366.x [doi].

30. Tholey MJ, Swain MV, Thiel N. Thermal gradients and residual stresses in veneered Y-TZP frameworks. Dent Mater. 2011;27(11):1102-1110. doi: 10.1016/j.dental.2011.08.001 [doi].

31. Lins L, Bemfica V, Queiroz C, Canabarro A. In vitro evaluation of the internal and marginal misfit of CAD/CAM zirconia copings. J Prosthet Dent. 2015;113(3):205211. doi: 10.1016/j.prosdent.2014.09.010 [doi].

32. Koenig V, Vanheusden AJ, Le Goff SO, Mainjot AK. Clinical risk factors related to failures with zirconia-based restorations: An up to 9-year retrospective study. J Dent. 2013;41(12):1164-1174. doi: 10.1016/j.jdent.2013.10.009 [doi].

33. Sailer I, Makarov NA, Thoma DS, Zwahlen M, Pjetursson BE. All-ceramic or metalceramic tooth-supported fixed dental prostheses (FDPs)? A systematic review of the survival and complication rates. part I: Single crowns (SCs). Dent Mater. 2015;31(6):603-623. doi: 10.1016/j.dental.2015.02.011 [doi].

34. Belli R, Frankenberger R, Appelt A, et al. Thermal-induced residual stresses affect the lifetime of zirconia-veneer crowns. Dent Mater. 2013;29(2):181-190. doi: 10.1016/j.dental.2012.11.015 [doi].

35. Meira JB, Reis BR, Tanaka CB, et al. Residual stresses in Y-TZP crowns due to changes in the thermal contraction coefficient of veneers. Dent Mater. 2013;29(5):594-601. doi: 10.1016/j.dental.2013.03.012 [doi]. 
36. Paula VG, Lorenzoni FC, Bonfante EA, Silva NR, Thompson VP, Bonfante G. Slow cooling protocol improves fatigue life of zirconia crowns. Dent Mater. 2015;31(2):77-87. doi: 10.1016/j.dental.2014.10.005 [doi].

37. Nordahl N, Vult von Steyern P, Larsson C. Fracture strength of ceramic monolithic crown systems of different thickness. J Oral Sci. 2015;57(3):255-261. doi: 10.2334/josnusd.57.255 [doi].

38. Nakamura K, Harada A, Inagaki R, et al. Fracture resistance of monolithic zirconia molar crowns with reduced thickness. Acta Odontol Scand. 2015;73(8):602-608. doi: 10.3109/00016357.2015.1007479 [doi].

39. Nakamura K, Harada A, Kanno T, et al. The influence of low-temperature degradation and cyclic loading on the fracture resistance of monolithic zirconia molar crowns. J Mech Behav Biomed Mater. 2015;47:49-56. doi: 10.1016/j.jmbbm.2015.03.007 [doi].

40. Goodacre CJ, Campagni WV, Aquilino SA. Tooth preparations for complete crowns: An art form based on scientific principles. J Prosthet Dent. 2001;85(4):363-376. doi: S0022-3913(01)57751-3 [pii].

41. Rosenstiel L, Fujimoto. Contemporary fixed prosthodontics. 4th ed. St. Louis, Missouri: Rudolph, Penny; 2006.

42. Wataha JC. Biocompatibility of dental casting alloys: A review. J Prosthet Dent. 2000;83(2):223-234. doi: S0022391300005655 [pii].

43. Raigrodski AJ, Hillstead MB, Meng GK, Chung KH. Survival and complications of zirconia-based fixed dental prostheses: A systematic review. J Prosthet Dent. 2012;107(3):170-177. doi: 10.1016/S0022-3913(12)60051-1 [doi].

44. Shi JY, Li X, Ni J, Zhu ZY. Clinical evaluation and patient satisfaction of single zirconia-based and high-noble alloy porcelain-fused-to-metal crowns in the esthetic area: A retrospective cohort study. J Prosthodont. 2015. doi: 10.1111/jopr.12344 [doi].

45. Pine CM, Pitts NB, Steele JG, Nunn JN, Treasure E. Dental restorations in adults in the UK in 1998 and implications for the future. Br Dent J. 2001;190(1):4-8.

46. Wilson NA, Whitehead SA, Mjor IA, Wilson NH. Reasons for the placement and replacement of crowns in general dental practice. Prim Dent Care. 2003;10(2):5359.

47. Schwartz NL, Whitsett LD, Berry TG, Stewart JL. Unserviceable crowns and fixed partial dentures: Life-span and causes for loss of serviceability. J Am Dent Assoc. 
1970;81(6):1395-1401.

48. Walton JN, Gardner FM, Agar JR. A survey of crown and fixed partial denture failures: Length of service and reasons for replacement. J Prosthet Dent. 1986;56(4):416-421.

49. Goodacre CJ, Bernal G, Rungcharassaeng K, Kan JY. Clinical complications in fixed prosthodontics. J Prosthet Dent. 2003;90(1):31-41. doi: $10.1016 /$ S0022391303002142 [doi].

50. Libby G, Arcuri MR, LaVelle WE, Hebl L. Longevity of fixed partial dentures. $J$ Prosthet Dent. 1997;78(2):127-131. doi: S0022-3913(97)70115-X [pii].

51. Walton TR. The up to 25-year survival and clinical performance of 2,340 high goldbased metal-ceramic single crowns. Int J Prosthodont. 2013;26(2):151-160. doi: 10.11607/ijp.3136 [doi].

52. Scurria MS, Bader JD, Shugars DA. Meta-analysis of fixed partial denture survival: Prostheses and abutments. J Prosthet Dent. 1998;79(4):459-464. doi: S00223913(98)70162-3 [pii].

53. Pjetursson BE, Lang NP. Prosthetic treatment planning on the basis of scientific evidence. J Oral Rehabil. 2008;35 Suppl 1:72-79. doi: 10.1111/j.13652842.2007.01824.x [doi].

54. Siegel SC, von Fraunhofer JA. Cutting efficiency of three diamond bur grit sizes. $J$ Am Dent Assoc. 2000;131(12):1706-1710.

55. Siegel SC, von Fraunhofer JA. Dental cutting with diamond burs: Heavy-handed or light-touch? J Prosthodont. 1999;8(1):3-9. doi: S1059941X99000133 [pii].

56. Ercoli C, Rotella M, Funkenbusch PD, Russell S, Feng C. In vitro comparison of the cutting efficiency and temperature production of ten different rotary cutting instruments. part II: Electric handpiece and comparison with turbine. J Prosthet Dent. 2009;101(5):319-331. doi: 10.1016/S0022-3913(09)60064-0 [doi].

57. Eikenberg SL. Comparison of the cutting efficiencies of electric motor and air turbine dental handpieces. Gen Dent. 2001;49(2):199-204.

58. Choi C, Driscoll CF, Romberg E. Comparison of cutting efficiencies between electric and air-turbine dental handpieces. J Prosthet Dent. 2010;103(2):101 <last_page> 107. doi: 10.1016/S0022-3913(10)60013-3.

59. Rotella M, Ercoli C, Funkenbusch PD, Russell S, Feng C. Performance of single-use and multiuse diamond rotary cutting instruments with turbine and electric handpieces. J Prosthet Dent. 2014;111(1):56-63. doi: 
10.1016/j.prosdent.2013.06.003 [doi].

60. von Fraunhofer JA, Siegel SC, Feldman S. Handpiece coolant flow rates and dental cutting. Oper Dent. 2000;25(6):544-548.

61. Siegel SC, von Fraunhofer JA. Dental burs--what bur for which application? A survey of dental schools. J Prosthodont. 1999;8(4):258-263. doi: S1059941X99000054 [pii].

62. Sharma S, Shankar R, Srinivas K. An epidemiological study on the selection, usage and disposal of dental burs among the dental practioner's. J Clin Diagn Res. 2014;8(1):250-254. doi: 10.7860/JCDR/2014/5916.3946 [doi].

63. DuraBraze different by design. . 2014.

64. Komet USA catalog. . 2010.

65. Blue DS, Griggs JA, Woody RD, Miller BH. Effects of bur abrasive particle size and abutment composition on preparation of ceramic implant abutments. The Journal of prosthetic dentistry. 2003;90(3):247.

66. Harada K, Shinya A, Gomi H, Hatano Y, Shinya A, Raigrodski AJ. Effect of accelerated aging on the fracture toughness of zirconias. J Prosthet Dent. 2015. doi: S0022-3913(15)00507-7 [pii].

67. Flinn BD, deGroot DA, Mancl LA, Raigrodski AJ. Accelerated aging characteristics of three yttria-stabilized tetragonal zirconia polycrystalline dental materials. $J$ Prosthet Dent. 2012;108(4):223-230. doi: 10.1016/S0022-3913(12)60166-8 [doi].

68. Kvam K, Karlsson S. Solubility and strength of zirconia-based dental materials after artificial aging. J Prosthet Dent. 2013;110(4):281-287. doi: 10.1016/S00223913(13)60377-7 [doi].

69. Cotes C, Arata A, Melo RM, Bottino MA, Machado JPB, Souza ROA. Effects of aging procedures on the topographic surface, structural stability, and mechanical strength of a ZrO2-based dental ceramic. Dental Materials. 2014;30(12):e396 <last_page> e404. doi: 10.1016/j.dental.2014.08.380.

70. Pereira GK, Venturini AB, Silvestri T, et al. Low-temperature degradation of Y-TZP ceramics: A systematic review and meta-analysis. J Mech Behav Biomed Mater. 2015;55:151-163. doi: S1751-6161(15)00390-2 [pii].

71. Pereira G, Amaral M, Cesar PF, Bottino MC, Kleverlaan CJ, Valandro LF. Effect of low-temperature aging on the mechanical behavior of ground Y-TZP. J Mech Behav Biomed Mater. 2015;45:183-192. doi: 10.1016/j.jmbbm.2014.12.009 [doi]. 
72. Kosmac T, Oblak C, Jevnikar P, Funduk N, Marion L. The effect of surface grinding and sandblasting on flexural strength and reliability of Y-TZP zirconia ceramic. Dent Mater. 1999;15(6):426-433. doi: S0109564199000706 [pii].

73. Cranska JP. Laser removal of all-ceramic restorations. solving a difficult clinical challenge. Dent Today. 2015;34(3):110-113.

74. Rechmann P, Buu NC, Rechmann BM, Finzen FC. Laser all-ceramic crown removala laboratory proof-of-principle study-phase 2 crown debonding time. Lasers Surg Med. 2014;46(8):636-643. doi: 10.1002/lsm.22280 [doi]. 


\section{Curriculum Vitae}

Shelby A. Alexander, D.D.S.

513 Pinnacle Heights Drive, Morgantown, WV 26505 | (856) 625-0961|

sfaltot@mix.wvu.edu

EDUCATION

West Virginia University, Morgantown, WV

[M.S. in Prosthodontics]- expected graduation May 2016

2013-Present

West Virginia University, Morgantown, WV

Doctor of Dental Surgery

2009-2013

Summa Cum Laude

West Virginia Wesleyan College, Buckhannon, WV

B.S. in Biology

2003-2007

Licensure

West Virginia Board of Dentistry

2013-Present

\section{MEMBERSHIPS}

American College of Prosthodontists

Academy of Osseointegration

Delta Sigma Delta

\section{LANGUAGES}

[English - native language] 